\title{
ON A MODEL OF ROTATING SUPERFLUIDS
}

\author{
SylVia SERFATY ${ }^{1}$
}

\begin{abstract}
We consider an energy-functional describing rotating superfluids at a rotating velocity $\omega$, and prove similar results as for the Ginzburg-Landau functional of superconductivity: mainly the existence of branches of solutions with vortices, the existence of a critical $\omega$ above which energyminimizers have vortices, evaluations of the minimal energy as a function of $\omega$, and the derivation of a limiting free-boundary problem.
\end{abstract}

Mathematics Subject Classification. 35Q99, 35J60, 35J50, 35B40, 35B25.

Received February 1, 2000. Revised November 28, 2000.

\section{INTRODUCTION}

\subsection{The energy functional}

The aim of this paper is to study a question that was asked by Pomeau, concerning a model of rotating superfluids. The evolution of a superfluid, such as superfluid helium II at zero temperature, is generally modelled (after some rescaling) by the following nonlinear Schrödinger equation, called the Gross-Pitaevskii equation:

$$
-i \hbar \frac{\partial u}{\partial t}=\hbar^{2} \Delta u+u\left(1-|u|^{2}\right) .
$$

The Gross-Pitaevskii equation is also used to model the evolution of Bose-Einstein condensates. Here $u$ is a complex-valued function characterizing the local state of the superfluid (it is a pseudo wave-function and $0 \leq|u| \leq 1$ ). If the superfluid is in a cylindrical bucket of two-dimensional section $\Omega$, smooth, bounded and simply connected, and rotating around a vertical axis at the angular velocity $\omega$; then, its energy, written in the rotating frame, taking into account the Coriolis force, is

$$
\int_{\Omega} \hbar^{2}|\nabla u+i u \omega \times x|^{2}+\frac{1}{2}\left(1-|u|^{2}\right)^{2}
$$

supplemented with the boundary condition $u=0$ on $\partial \Omega$. Here $x=\left(x_{1}, x_{2}\right) \in \Omega$ with the origin set at the rotation axis, and $\times$ is the vectorial product in $\mathbb{R}^{3}$. By $i u \omega \times x$ we mean the complex-valued vector $i u \omega\left(x_{2}, x_{1}\right)$, then considering $\omega$ as a positive real number.

Keywords and phrases: Vortices, Gross-Pitaevskii equations, superfluids.

1 CMLA, École Normale Supérieure de Cachan, 61 avenue du Président Wilson, 94235 Cachan Cedex, France;

e-mail: serfaty@cmla.ens-cachan.fr 
This model could also serve to describe Bose-Einstein condensates, whose evolution is given by the GrossPitaevskii equation. For a rotating Bose-Einstein condensate trapped in a harmonic potential, a more realistic model includes a term $\int_{\Omega}\left(a(x)-|u|^{2}\right)^{2}$ where $a(x)$ is a quadratic function vanishing on $\partial \Omega$ instead of $\int_{\Omega}\left(1-|u|^{2}\right)^{2}$, (see [9] and [2]), but would also lead to the same kind of analysis.

We replace the study of (1.1) by the study of

$$
J(u)=\frac{1}{2} \int_{\Omega}|\nabla u+i u \omega \times x|^{2}+\frac{1}{2 \varepsilon^{2}}\left(1-|u|^{2}\right)^{2},
$$

over $H_{0}^{1}(\Omega, \mathbb{C})$, where $\varepsilon$ is a small parameter. If we expand the first term, we obtain

$$
J(u)=\frac{1}{2} \int_{\Omega}|\nabla u|^{2}+\frac{1}{2 \varepsilon^{2}}\left(1-|u|^{2}\right)^{2}+\frac{\omega^{2}}{2} \int_{\Omega}|u|^{2}|x|^{2}+\omega \int_{\Omega}\left(i u, x_{2} u_{x_{1}}-x_{1} u_{x_{2}}\right) .
$$

Here (.,.) denotes the scalar product in $\mathbb{R}^{2}$, where complex numbers are seen as belonging to $\mathbb{R}^{2}$. Another minimization problem which can be considered to derive this is the following: minimize a Hamiltonian of the form

$$
H=\frac{1}{2} \int_{\Omega}|\nabla u|^{2}+\frac{1}{2 \varepsilon^{2}}\left(1-|u|^{2}\right)^{2}
$$

where $u \in H_{0}^{1}(\Omega, \mathbb{C})$, with fixed angular momentum

$$
M=\int_{\Omega}(i u, x \times \nabla u)=\int_{\Omega}\left(i u, x_{1} u_{x_{2}}-x_{2} u_{x_{1}}\right) .
$$

(The Hamiltonian $H$ and the momentum $M$ are quantities that are conserved in time for the evolution of the type (1.1).) Using a Lagrange multiplier $\lambda$, this is equivalent to minimizing

$$
H-\lambda M=\frac{1}{2} \int_{\Omega}|\nabla u|^{2}+\frac{1}{2 \varepsilon^{2}}\left(1-|u|^{2}\right)^{2}-\lambda \int_{\Omega}\left(i u, x_{1} u_{x_{2}}-x_{2} u_{x_{1}}\right) .
$$

Up to the term $\frac{1}{2} \omega^{2} \int_{\Omega}|u|^{2}|x|^{2}$, this is the same expression as (1.3) for $\omega=\lambda$. Thus, the rotation velocity $\omega$ can be seen as the Lagrange multiplier in the previous problem. On the other hand, we shall see that if $\omega$ is sufficiently small compared to $\frac{1}{\varepsilon}$, the term $\frac{1}{2} \omega^{2} \int_{\Omega}|u|^{2}|x|^{2}$ is of lower order in the energy, hence can be neglected, since, up to slight adjustments in our proofs, it would lead to the same qualitative results.

Another question that physicists consider is to minimize an energy of the type $J$ or $H-\lambda M$ with a fixed "number of particles" $N=\int_{\Omega}|u|^{2}$. Again, this can be taken into account through a Lagrange multiplier. It adds a term which is also negligible when $\varepsilon$ is small and $\omega$ not too large. Thus, we reduce to the study of $J$ given by the expressions (1.2) or (1.3).

As already mentioned, $\varepsilon$ is a small parameter, we will actually make it tend to zero. This corresponds to the case where the characteristic scale of the phenomenon $\varepsilon$, is small compared to the scale of the domain, which is relevant for usual sizes of domains, and is a limit often considered by physicists (see for example [10]). In the physics of Bose-Einstein condensates, $\varepsilon$ small corresponds to the "Thomas-Fermi" approximation (see [2,9]).

The question is, of course, to find steady states (or critical points) for this energy in the rotating frame, and to describe them. The main feature of rotating superfluids is that, for certain velocities, they exhibit vortices: $u$ has some isolated zeros in $\Omega$, and $\frac{u}{|u|}$ has a nonvanishing (topological) degree around these zeros. More precisely, consider $a$ a point where $u$ vanishes and $r>0$ small such that $u$ does not vanish on $\partial B(a, r)$, then $\frac{u}{|u|}$ is a mapping from $\partial B(a, r)$ to $S^{1}$, hence it has a topological degree, or winding number (which is the number of turns of the phase of $u$ ). This is what is called the degree of the isolated zero. The characteristic scale of the phenomenon is thus $\varepsilon$, the scale of a vortex. In experiments, there can be up to thousands of vortices in the domain. For more details on the physical aspects, one can refer to the physical litterature ([10,24] for example). 
This behaviour of superfluids is very similar to the behaviour of superconductors in an external magnetic field. Actually, we prove here that there is a total analogy between this model and the Ginzburg-Landau model of superconductivity, and that we can adjust our results on the Ginzburg-Landau energy to this functional. The Ginzburg-Landau functional for superconductors is

$$
G(u, A)=\int_{\Omega} \frac{1}{2}|\nabla u-i A u|^{2}+\frac{1}{2}\left|\operatorname{curl} A-h_{\mathrm{ex}}\right|^{2}+\frac{1}{4 \varepsilon^{2}}\left(1-|u|^{2}\right)^{2},
$$

where $h_{\mathrm{ex}}$ is the intensity of the applied magnetic field, $A=\left(A_{1}, A_{2}\right) \in \mathbb{R}^{2}$ is the vector potential of the magnetic field, and $h=\operatorname{curl} A$ the induced magnetic field in the material. The first term $\int_{\Omega}|\nabla u+i u \omega \times x|^{2}$ is very similar to the term $\int_{\Omega}|\nabla u-i A u|^{2}$ in the Ginzburg-Landau functional. Actually, $J$ is even simpler, it only depends on one function, and, as we shall see, the role of the external field $h_{\mathrm{ex}}$ is replaced by the angular velocity $\omega$.

In [16-18,20-22], we studied in details the functional (1.4) and its minimizers, and proved that they exhibited a vortex-structure when $H_{c_{1}} \leq h_{\mathrm{ex}} \leq H_{c_{2}}$, where $H_{c_{1}}$ and $H_{c_{2}}$ are critical values depending on $\varepsilon$. Here, we adjust these results and obtain very similar ones.

Let us emphasize that the main difference between the two problems is the boundary condition: here $u=0$ on $\partial \Omega$ whereas, for Ginzburg-Landau, all functions in $H^{1}$ were admissible, so no boundary conditions were imposed. This condition $u=0$ induces a cost of $\frac{C}{\varepsilon}$ at least in the energy, because $u$ has to be small on a layer of size of the order of $\varepsilon$ near $\partial \Omega$. This cost is very large compared to the Ginzburg-Landau energy $G$. Hence, if we make comparisons with test maps, all the fine information on the behaviour of $u$ in $\Omega$ will be hidden by the energetic cost of the boundary layer. The method for solving this problem was suggested to us by Shafrir, and is one that has been introduced by Lassoued and Mironescu [13] and also used by André and Shafrir in [4]. It consists in dividing $u$ by $\rho$, the real-valued function which vanishes at $\partial \Omega$ and minimizes $J$ over the space of real-valued functions. Then, we can prove that $J$ splits as

$$
J(u)=J(\rho)+\int_{\Omega} \frac{\rho^{2}}{2}|\nabla v|^{2}+\frac{\rho^{4}}{4 \varepsilon^{2}}\left(1-|v|^{2}\right)^{2}+\omega \int_{\Omega} \rho^{2}\left(i v, x_{2} v_{x_{1}}-x_{1} v_{x_{2}}\right),
$$

where $v=\frac{u}{\rho}$. J $J(\rho)$ contains the boundary layer contribution while $J(u)-J(\rho) \ll J(\rho)$ can be studied as the Ginzburg-Landau functional. Let us emphasize again that the ideas of the results are not new, but borrowed from those of $[4,13,16-18,20,21]$, and that this paper consists in showing that these ideas remain valid and can be adjusted to this new problem.

\subsection{Notations}

We study $J$ on $H_{0}^{1}(\Omega, \mathbb{C})$. Critical points of $J$ are solutions of the following associated Euler equation:

$$
\text { (G.P.) }\left\{\begin{array}{l}
-\Delta u=\frac{u}{\varepsilon^{2}}\left(1-|u|^{2}\right)+2 i \nabla u . \omega \times x-\omega^{2} r^{2} u \text { in } \Omega \\
u=0 \text { on } \partial \Omega
\end{array}\right.
$$

that we call the Gross-Pitaevskii equation. By the maximum principle, a solution $u$ of (G.P.) satisfies $|u| \leq 1$. We write $x=\left(x_{1}, x_{2}\right)$ and $r=|x|, \times$ is the vector product in $\mathbb{R}^{3}$ while $($,$) is the scalar product on \mathbb{R}^{2}$. $\omega$ denotes the rotation-vector perpendicular to $\Omega$ in $\mathbb{R}^{3}$ in the expression $\omega \times x$, otherwise its norm. We write $\nabla^{\perp} f=\left(-f_{x_{2}}, f_{x_{1}}\right)$. F will denote the functional studied in [5], i.e.

$$
F(u)=\frac{1}{2} \int_{\Omega}|\nabla u|^{2}+\frac{1}{2 \varepsilon^{2}}\left(1-|u|^{2}\right)^{2} .
$$

For any subset $V \subset \Omega, J_{V}$ or $F_{V}$ will denote the energy-functionals restricted to $V$. The domain $D_{\mathcal{M}}$ over which we perform a local minimization of $J$, corresponds roughly to the $u \in H_{0}^{1}$ for which $F(u) \leq \mathcal{M}|\log \varepsilon|$. $\mathcal{R}$ will denote the space of Radon measures on $\Omega$. 


\subsection{Statement of the results of existence of branches of solutions}

We prove the following results, where the notion of "vortex" will be specified later. In all the paper, $\omega$ is considered as a function of $\varepsilon$ such that $\omega \varepsilon \rightarrow 0$ as $\varepsilon \rightarrow 0$, and $C$ denotes some positive constant, independent of $\varepsilon$.

Theorem 1. Suppose $\Omega=B_{R}=B(0, R)$. Defining a rotation velocity $\omega_{1}$ by

$$
\omega_{1}=\frac{|\log \varepsilon|}{R^{2}}
$$

there exist $k(\varepsilon)=O(1), k^{\prime}(\varepsilon)=O(|\log | \log \varepsilon||)$, and $\varepsilon_{0}(\mathcal{M})$ such that for $\varepsilon<\varepsilon_{0}$, the following holds:

- if and only if $\omega \leq \omega_{1}-k(\varepsilon)$, the minimum of $J$ is $J(\rho)-o(1)$ and if $\omega \leq \omega_{1}-k^{\prime}(\varepsilon)$ any minimizer is vortex-less;

- if $\omega_{1}+k(\varepsilon) \leq \omega \leq \omega_{1}+O(1)$, there exists a minimizer of $J$ over $D_{\mathcal{M}}$ which is a solution of (G.P.). In addition, it has exactly one vortex a of degree one, and $|a| \rightarrow 0$ as $\varepsilon \rightarrow 0$.

This theorem which is similar to Theorem 1 of [16], shows that there exists a critical value of $\omega$ above which vortices become energetically favourable. The expression of $\omega_{1}$, equivalent to that of $H_{c_{1}}$ in [16], is an explicit function of the size of the domain, and corresponds to the expressions found in physics literature (see [10]).

Theorem 2. Suppose $\Omega=B_{R}$, and $\omega$ is any function of $\varepsilon$ such that $\omega \rightarrow+\infty$ as $\varepsilon \rightarrow 0$, and $\omega \leq C \varepsilon^{-\alpha}$ for some small $\alpha>0$; then $\forall n \in \mathbb{N}^{*}$ such that $n<\frac{\mathcal{M}}{\pi}$, and $\forall \varepsilon<\varepsilon_{0}$, there exists a branch of stable solutions of (G.P.) such that:

1) $u$ has exactly $n$ vortices of degree 1 , located at $a_{i}^{\varepsilon}$.

2) $\left|a_{i}^{\varepsilon}\right| \rightarrow 0$ as $\varepsilon \rightarrow 0$, and if we set $\tilde{a_{i}}=a_{i} \sqrt{\omega}$, the $\tilde{a}_{i}$ 's tend to minimize

$$
w\left(x_{1}, \cdots, x_{n}\right)=-\pi \sum_{i \neq j} \log \left|x_{i}-x_{j}\right|+\pi \sum_{i}\left|x_{i}\right|^{2}
$$

so that $\left|a_{i}\right| \leq \frac{C}{\sqrt{\omega}}$, and $\left|a_{i}-a_{j}\right| \geq \frac{C}{\sqrt{\omega}}$.

3)

$$
J(u)=J(\rho)+\pi n\left(|\log \varepsilon|-R^{2} \omega\right)+\frac{\pi}{2}\left(n^{2}-n\right) \log \omega+w\left(\tilde{a_{1}}, \cdots, \tilde{a_{n}}\right)+Q_{n}+o(1) .
$$

The solution with $n$ vortices minimizes $J$ in $D_{\mathcal{M}}$ exactly for $\omega_{n} \leq \omega \leq \omega_{n+1}$, where $\omega_{n}$ has an expression of the form

$$
\omega_{n}=\frac{|\log \varepsilon|}{R^{2}}+\frac{n-1}{R^{2}}|\log | \log \varepsilon||+O(1) .
$$

The result can also be reformulated as follows: $\forall n \in \mathbb{N}$, there exists $\varepsilon_{0}(n)$ such that $\forall \varepsilon<\varepsilon_{0}(n)$, there exists a branch of stable solutions of (G.P.) satisfying 1), 2) and 3).

This theorem is the analogue of Theorem 2 of [18]. It proves, in the case of a disc, the existence of branches of stable solutions with $n$ vortices of degree 1 . These solutions coexist for a wide range of $\omega$, their energy follows a simple explicit formula. In addition, they are globally minimizing, i.e. they achieve the minimum over all $H_{0}^{1}$, for $\omega_{n} \leq \omega \leq \omega_{n+1}$; this has been proved for the Ginzburg-Landau energy in a forthcoming paper [23].

What seems most interesting to us is the minimization of $w$ : this says that we can replace the minimization of $J$ over $H_{0}^{1}$ by the minimization of the explicit function $w$ over $\Omega^{n}$. After rescaling, the positions of the vortices of our branches of solutions tend to minimize $w$. Then, the natural question is to ask what minimizers of $w$ look like. This is not so easy to calculate. Shafrir and Gueron have worked on this problem (see [11]). They prove that for $n \leq 6$, the regular polygons centered at the origin are local (and very likely) global minimizers, for $4 \leq n \leq 6$ there are other stable critical shapes: the regular "stars" which are regular polygons centered 
at the origin plus the origin. For $7 \leq n \leq 11$, they are again local minimizers (and probably global). For higher $n$, numerically, the minimizers look like series of concentric polygons and then triangular lattices, first concentrated around the origin, then scattered all over $\Omega$, as $n$ increases. Observations have been made (since the 70's) on the vortices in rotating superfluid helium II, which show pictures of vortices which are exactly the ones described for the minimizers of $w$ : i.e. regular polygons, stars, lattices. One can refer to [24] for pictures.

Thus, our results agree with the physical observations and theoretical predictions (see [10] for superfluids), and particularly with those found in $[8,9]$ on Bose-Einstein condensates. Moreover, they state precise values of the $\omega_{n}$ for which the $n$-th vortex becomes energetically favourable, which seems to be a new result, they say that the vortices are concentrated around 0 at a scale $\frac{C}{\sqrt{\omega}}$ and prove the multiplicity of stable solutions for a given $\omega$ around $\omega_{1}$.

\subsection{Methods of the proofs}

As the proofs are borrowed from other papers, we only explain their main step s. For Theorems 1 and 2 , let us just say that the method consists in splitting $J$ as (1.3) and then splitting $J-J(\rho)$ similarly as in [16]. The term

can be replaced by

$$
\int_{B_{R}} \frac{\rho^{2}}{2}|\nabla v|^{2}+\frac{\rho^{4}}{4 \varepsilon^{2}}\left(1-|v|^{2}\right)^{2}
$$

$$
\int_{B_{R^{\prime}}} \frac{1}{2}|\nabla v|^{2}+\frac{1}{4 \varepsilon^{2}}\left(1-|v|^{2}\right)^{2}=F_{B_{R^{\prime}}}(v)
$$

where $R^{\prime}=R-\varepsilon^{\beta}(0<\beta<1)$ and $F$ is the functional studied in [5]. Then, we prove that if $u$ is a configuration with a bounded number of vortices $a_{i}$ of degree $d_{i}$, then the angular momentum $M$ can be expressed as:

$$
M=\int_{B_{R}}\left(i u, x_{1} u_{x_{2}}-x_{2} u_{x_{1}}\right) \simeq-\int_{B_{R}}(i v, d v \wedge d X) \simeq-2 \pi \sum_{i} d_{i} X\left(a_{i}\right) \simeq \pi \sum_{i} d_{i}\left(R^{2}-\left|a_{i}\right|^{2}\right),
$$

where $X=\frac{|x|^{2}-R^{2}}{2}$. Here $X$ plays the same role as $\xi$ in [16-18], hence we can perform the same analysis to evaluate the cost and gain of each vortex, and see that vortices will tend to the point of minimum of $X$ (which is the origin). To find our branches of $n$-vortices solutions, we perform a local minimization exactly as in [18], over domains of the type

$$
U_{n}=\left\{u \in H_{0}^{1}\left(B_{R}, \mathbb{C}\right) / n|\log \varepsilon|<F\left(\frac{u}{\rho}\right)<\left(n+\frac{1}{2}\right)|\log \varepsilon|\right\}
$$

and prove that it yields a solution of (G.P.) which has $n$ vortices.

\subsection{Statement of the results on global minimizers}

The following results are the analogues of those of [21] and [22] on the Ginzburg-Landau functional.

We assume that $\omega(\varepsilon)$ is such that $\omega \ll \frac{1}{\varepsilon^{2}}$ and that

$$
\lambda=\lim _{\varepsilon \rightarrow 0} \frac{|\log \varepsilon|}{\omega}
$$

exists and is finite. Then, for any $\lambda$, we define the limiting functional $E$ as:

$$
E(f)=\frac{\lambda}{2} \int_{\Omega}|\Delta f+2|+\frac{1}{2} \int_{\Omega}|\nabla f|^{2},
$$


over

$$
\left\{f \in H_{0}^{1}(\Omega) / \Delta f+2 \in \mathcal{R}\right\}
$$

where $\mathcal{R}$ is the space of bounded Radon measures on $\omega$.

We study any family $\left(u_{\varepsilon}\right)$ of global minimizers of $J$ over $H_{0}^{1}(\Omega)$. Such a $u_{\varepsilon}$ is solution of (G.P.), therefore one can check that it satisfies

$$
\operatorname{div}\left((i u, \nabla u)-\omega \nabla^{\perp} X\right)=0
$$

where $X=\frac{|x|^{2}}{2}$. We will see that we can find a unique $U_{\varepsilon} \in H_{0}^{1}$ such that

$$
\nabla^{\perp} U=(i u, \nabla u)-\omega \nabla^{\perp} X
$$

This equation is the analogue of the second Ginzburg-Landau equation. It yields a relevant quantity $U$ which plays the same role as the induced magnetic field $h$ for Ginzburg-Landau. We shall see how $U$ is related to the total vorticity of $u$.

Theorem 3. 1) Assume $\lambda$ exists and is finite, $\omega \ll \frac{1}{\varepsilon^{2}}, u_{\varepsilon}$ minimizes $J$ and $U_{\varepsilon}$ is associated to $u_{\varepsilon}$ by (1.10). Then, as $\varepsilon \rightarrow 0$,

$$
\frac{U_{\varepsilon}}{\omega} \rightarrow U_{*} \quad \text { weakly in } H_{0}^{1}(\Omega)
$$

where $U_{*}$ is the unique minimizer of $E$, and solution of the following obstacle problem:

$$
\begin{cases}U_{*}=0 & \text { on } \partial \Omega \\ U_{*} \leq \frac{\lambda}{2} & \text { in } \Omega \\ \left(\Delta U_{*}+2\right)\left(U_{*}-\frac{\lambda}{2}\right)=0 & \text { in } \Omega \\ \Delta U_{*}+2 \geq 0 . & \end{cases}
$$

In addition $U_{*} \in C^{1, \alpha}(\Omega), \forall \alpha<1$. Moreover,

$$
\min J \sim_{\varepsilon \rightarrow 0} F(\rho)+\omega^{2} E\left(h_{*}\right)
$$

2) If $\lambda=0$, then $U_{*}=0$, and the convergence is strong in $H_{0}^{1}$. If $\lambda>0$, for $\varepsilon<\varepsilon_{0}$, we can find a family of balls $\left(B_{i}\right)_{i \in I_{\varepsilon}}=\left(B\left(a_{i}, r_{i}\right)\right)_{i \in I_{\varepsilon}}$ such that

$$
\begin{aligned}
& \left\{x, \operatorname{dist}(x, \partial \Omega) \geq \varepsilon^{\beta} /|| v|(x)-1| \geq \frac{1}{|\log \varepsilon|}\right\} \subset \cup_{i \in I_{\varepsilon}} B_{i} \\
& \sum_{i \in I_{\varepsilon}} r_{i} \leq \frac{1}{|\log \varepsilon|^{6}}, \\
& \forall i \in I_{\varepsilon}, \quad \frac{1}{2} \int_{B_{i}}|\nabla U|^{2} \geq \pi\left|d_{i}\right||\log \varepsilon|(1-o(1))
\end{aligned}
$$

where $d_{i}=\operatorname{deg}\left(u, \partial B_{i}\right)$.

For any such family, if we define $\mu_{\varepsilon}=\frac{2 \pi}{\omega} \sum_{i \in I_{\varepsilon}} d_{i} \delta_{a_{i}}$, we have

$$
\mu_{\varepsilon} \rightarrow \mu_{*}=\Delta U_{*}+2
$$


and

in the sense of measures.

$$
\frac{2 \pi}{\omega} \sum_{i \in I_{\varepsilon}}\left|d_{i}\right| \delta_{a_{i}} \rightarrow \mu_{*}
$$

3) If we set $\mathcal{U}_{\lambda}=\left\{x \in \Omega / U_{*}(x)=\frac{\lambda}{2}\right\}$, we have $\mu_{*}=2 \mathbf{1}_{\mathcal{U}_{\lambda}}$, where $\mathbf{1}_{\mathcal{U}_{\lambda}}$ denotes the characteristic function of $\mathcal{U}_{\lambda} \cdot \mathcal{U}_{\lambda}=\varnothing \Leftrightarrow \lambda \geq 2 \max \xi_{0}$ where $\xi_{0}$ is the solution of

$$
\begin{cases}-\Delta \xi_{0}=2 & \text { in } \Omega \\ \xi_{0}=0 & \text { on } \partial \Omega\end{cases}
$$

This theorem is mostly relevant in the intermediate case $\omega=O(|\log \varepsilon|)$ corresponding to $\lambda>0$. We then isolate the zeroes of $u_{\varepsilon}$ (which are not too close to $\partial \Omega$ ) in vortex balls $B_{i}$ and define a vorticity measure $\mu_{\varepsilon}$, proved to be closely related to $U_{\varepsilon} \cdot \mu_{\varepsilon}$ converges weakly to $\mu_{*}$ which is a uniform measure of density 2 on a subset $\mathcal{U}_{\lambda}$ of $\Omega$. Thus, qualitatively, we expect $u_{\varepsilon}$ to have vortices of positive degrees, regularly scattered over $\mathcal{U}_{\lambda}$ with a density $\sim 2 \omega$ when $\varepsilon$ is sufficiently small. $\mathcal{U}_{\lambda}$ is determined by (1.7) which is a free boundary problem. It is a classical obstacle problem (see [12]). If $\partial \mathcal{U}_{\lambda}$ is smooth (which is not always the case, but is the case at least for almost every value of $\lambda$ from a result of $[6]$ ), then (1.7) can be rewritten more simply:

$$
\begin{cases}U_{*}=0 & \text { on } \partial \Omega \\ -\Delta U_{*}=2 & \text { in } \Omega \backslash \mathcal{U}_{\lambda} \\ U_{*}=\frac{\lambda}{2} & \text { on } \partial \mathcal{U}_{\lambda} \\ \frac{\partial U_{*}}{\partial n}=0 & \text { on } \partial \mathcal{U}_{\lambda}\end{cases}
$$

The size of the vortex-region $\mathcal{U}_{\lambda}$ depends on $\lambda$. If $\lambda$ is very large (corresponding to small $\omega$ 's), then $\mathcal{U}_{\lambda}=\varnothing$. More precisely, if $\omega \leq \omega_{1} \sim \frac{|\log \varepsilon|}{2 \max \xi_{0}}$, then $\mathcal{U}_{\lambda}=\varnothing$, and following [20], we could have proved rigorously that $u_{\varepsilon}$ has no vortex in this case. Thus, some $\omega_{1} \sim \frac{|\log \varepsilon|}{2 \max \xi_{0}}$ or $\lambda=2 \max \xi_{0}$ corresponds to a critical value (first critical velocity), and is compatible with the result of Theorem 1 . Indeed, if $\Omega$ is a ball $B(0, R)$, then $\xi_{0}=\frac{R^{2}-|x|^{2}}{2}$, and thus $2 \max \xi_{0}=R^{2}$. This theorem generalizes the result of Theorem 1 to arbitrary simply connected geometries.

If $\omega \geq \omega_{1}$, then $\mathcal{U}_{\lambda}$ is nonempty and minimizers have vortices. $\mathcal{U}_{\lambda}$ increases as $\lambda$ decreases (i.e. as $\omega$ increases), until, for $\lambda=0$, corresponding to $\omega \gg|\log \varepsilon|, \mathcal{U}_{\lambda}=\Omega$, and the vortices fill all $\Omega$. The main difference compared to the result of [22] on the Ginzburg-Landau functional is that the limiting measure $\mu_{*}$ always has density 2 , whereas in Ginzburg-Landau it had a density $1-\frac{\lambda}{2}$, thus depending on $\lambda$ and on the applied field.

(1.12) provides an asymptotic expansion of the minimal energy, in which $F(\rho)$ carries the boundary layer cost of any configuration due to the boundary condition $u=0$. Indeed, $F(\rho)=\frac{1}{2} \int_{\Omega}|\nabla \rho|^{2}+\frac{1}{2 \varepsilon^{2}}\left(1-\rho^{2}\right)^{2}$ is of the order of $\frac{\sqrt{2}}{3 \varepsilon} l(\partial \Omega)$ as we shall see in Section 2 , and $F(\rho)=J(\rho)-\frac{1}{2} \int_{\Omega} \omega^{2} r^{2} \rho^{2}$, hence, as soon as $\omega^{2} \ll \frac{1}{\varepsilon}, F(\rho) \sim J(\rho)$ is the term of highest order in (1.12). In the case of $\omega \gg|\log \varepsilon|$ i.e. when $\lambda=0$, then this theorem only states that $\min J \sim F(\rho)$, and $\frac{U_{\varepsilon}}{\omega} \rightarrow 0$. We are in fact able to get more precise results (adjusted from [21]) in the following theorems:

Theorem 4. Assume $|\log \varepsilon| \ll \omega \ll \frac{1}{\varepsilon}$. Then

$$
J(\rho)-\int_{\Omega} \frac{\omega^{2}}{2} r^{2}+\omega|\Omega| \log \frac{1}{\varepsilon \sqrt{\omega}}(1-o(1)) \leq \min _{H_{0}^{1}(\Omega, \mathbb{C})} J \leq F(\rho)+\omega|\Omega| \log \frac{1}{\varepsilon \sqrt{\omega}}+O(\omega),
$$

where $|$.$| denotes the volume.$

If in addition $\omega \leq \frac{C}{\varepsilon^{4 / 5}}$, then

$$
\min _{H_{0}^{1}(\Omega, \mathbb{C})} J=F(\rho)+\omega|\Omega| \log \frac{1}{\varepsilon \sqrt{\omega}}(1+o(1))
$$


Theorem 5. Let $|\log \varepsilon| \ll \omega \leq \frac{C}{\varepsilon^{4 / 5}}$, and $u_{\varepsilon}$ be a corresponding minimizer of $J$. Then, for $\varepsilon<\varepsilon_{0}$, there exists a family of disjoint disks $\left(B_{i}^{\varepsilon}\right)$ with radii each less than $\frac{1}{\sqrt{\omega}}$ and sum less than $|\Omega| \sqrt{\omega}$, such that $\left|u_{\varepsilon}\right| \geq \frac{1}{2}$ on $\partial B_{i}^{\varepsilon}$ and, if $a_{i}^{\varepsilon}$ is the center of $B_{i}^{\varepsilon}$ and $d_{i}^{\varepsilon}=\operatorname{deg}\left(\frac{u_{\varepsilon}}{\left|u_{\varepsilon}\right|}, \partial B_{i}^{\varepsilon}\right)$, then

$$
\mu_{\varepsilon}=\frac{2 \pi}{\omega} \sum_{i} d_{i}^{\varepsilon} \delta_{a_{i}^{\varepsilon}} \underset{\varepsilon \rightarrow 0}{\longrightarrow} 2 d x
$$

in the weak sense of measures, where $d x$ is the Lebesgue measure on $\mathbb{R}^{2}$ restricted to $\Omega$.

Moreover,

$$
\pi \sum_{i}\left|d_{i}^{\varepsilon}\right| \simeq \pi \sum_{i} d_{i}^{\varepsilon} \simeq \omega|\Omega|
$$

and most of the vortex-energy is concentrated in the balls, i.e.

$$
J_{\Omega \backslash \cup_{i} B_{i}^{\varepsilon}}\left(u_{\varepsilon}\right)-F(\rho)=o\left(J\left(u_{\varepsilon}\right)-F(\rho)\right) .
$$

Of course, for any value of $\omega$, we have the trivial solution $u \equiv 0$ which has an energy $\frac{|\Omega|}{4 \varepsilon^{2}}$. We believe that, for $\omega$ higher than some critical value $\omega \geq \frac{C}{\varepsilon}$, it becomes minimal.

\section{The SPlitting of The Energy}

We introduce $\rho_{\varepsilon}$, for a general domain $\Omega$. It is defined to be the minimizer of the following problem:

$$
\min _{H_{0}^{1}(\Omega, \mathbb{R})} \frac{1}{2} \int_{\Omega}|\nabla \rho|^{2}+\frac{1}{2 \varepsilon^{2}}\left(1-\rho^{2}\right)^{2}+\omega^{2} r^{2} \rho^{2} .
$$

We will often drop the subscript and write $\rho$ instead of $\rho_{\varepsilon}$.

Lemma 2.1. $\rho_{\varepsilon}$ satisfies the following:

$$
\begin{aligned}
& \rho \in C^{\infty} \quad 0 \leq \rho_{\varepsilon} \leq 1, \quad|\nabla \rho| \leq \frac{C}{\varepsilon} \\
& \text { if } \Omega=B_{R}, \text { then } \rho \text { is radial and is a solution of }(G . P .), \\
& -\Delta \rho_{\varepsilon}+\omega^{2} r^{2} \rho_{\varepsilon}=\frac{1}{\varepsilon^{2}} \rho_{\varepsilon}\left(1-\rho_{\varepsilon}^{2}\right) \\
& 1-\rho_{\varepsilon} \leq C e^{-\frac{\delta(x)}{2 \varepsilon}}+O\left(\varepsilon^{2} \omega^{2}\right) \quad \text { where } \delta(x)=\operatorname{dist}(x, \partial \Omega) \\
& J(\rho) \leq \frac{\sqrt{2}}{3 \varepsilon} l(\partial \Omega)+\frac{\omega^{2}}{2} \int_{\Omega}|x|^{2}+O(1),
\end{aligned}
$$

where $l(\partial \Omega)$ denotes the length of $\partial \Omega$.

$$
\int_{\Omega}\left(1-\rho^{2}\right)^{2} \leq C\left(\varepsilon+\omega^{4} \varepsilon^{4}\right) .
$$

Proof. It is well-known since the work of Brezis and Oswald [7] that, as soon as $\varepsilon$ is small enough, there exists a positive minimizer for the functional (2.1), and that it is the only positive solution of

$$
\begin{cases}-\Delta \rho+\omega^{2} r^{2} \rho=\frac{1}{\varepsilon^{2}} \rho\left(1-\rho^{2}\right) & \text { in } \Omega \\ \rho=0 & \text { on } \partial \Omega .\end{cases}
$$


It is also standard that $\rho \leq 1$ and $|\nabla \rho| \leq \frac{C}{\varepsilon}$. If $\Omega=B_{R}$, the fact that $\rho$ is radial comes from the uniqueness of the positive solution, and the fact that $\rho$ satifies (G.P.) comes from (2.8).

We then prove (2.5). The proof is similar to that of Proposition 2.1 of [4].

Consider $x_{0}$ such that $\delta(x):=\operatorname{dist}\left(x_{0}, \partial \Omega\right)>K \varepsilon$, for some $K$ to be determined afterwards. Let $\phi_{1}$ be a positive eigenfunction corresponding to the first eigenvalue $\lambda_{1}$ of $-\Delta$ on $B(0,1)$ :

$$
\begin{cases}-\Delta \phi_{1}=\lambda_{1} \phi_{1} & \text { in } B(0,1) \\ \phi_{1}=0 & \text { on } \partial B(0,1)\end{cases}
$$

and satisfying $\phi_{1} \leq \frac{1}{2}$ on $B(0,1)$.

Let us write $\phi(x)=\phi_{1}\left(\frac{x-x_{0}}{K \varepsilon}\right)$, then $\Delta \phi=\frac{\lambda_{1}}{K^{2} \varepsilon^{2}} \phi$ on $B\left(x_{0}, K \varepsilon\right)$. If $K$ is chosen large enough (independent from $\varepsilon$ ), then

for small $\varepsilon$, since $\omega \varepsilon \rightarrow 0$. Hence,

$$
\frac{\lambda_{1}}{K^{2}} \phi \leq \phi\left(1-\phi^{2}\right)-\omega^{2} \varepsilon^{2} r^{2} \phi \quad \text { in } B\left(x_{0}, K \varepsilon\right),
$$

and thus $\phi$ is a subsolution for (2.8), implying

$$
-\Delta \phi \leq \frac{\phi}{\varepsilon^{2}}\left(1-\phi^{2}\right)-\omega^{2} r^{2} \phi
$$

$$
\rho \geq \phi \quad \text { in } B\left(x_{0}, K \varepsilon\right) .
$$

Therefore, there exists $1>a>0$, independent from $\varepsilon$, such that

$$
\rho \geq \phi \geq a>0 \text { in } B\left(x_{0}, K \varepsilon\right) .
$$

Hence

$$
\rho \geq a>0 \quad \text { in } \tilde{\Omega}:=\left\{x / \delta(x)>\frac{K \varepsilon}{2}\right\}
$$

Now, as in [4], it is enough to prove the estimate $(2.5)$ on $\tilde{\Omega}$. We prove it by using suitable subsolutions. Consider again $x_{0} \in \tilde{\Omega}$, and let $\mu=\operatorname{dist}\left(x_{0}, \partial \tilde{\Omega}\right)$. On $B\left(x_{0}, \mu\right)$, we consider $w_{1}$, the subsolution of [4], defined by:

As in [4], we have $w_{1} \geq a$ and

$$
w_{1}(\eta)=t h\left(t h^{-1} a+\frac{\mu^{2}-\eta^{2}}{3 \mu \varepsilon}\right), \quad \text { where } \eta=\left|x-x_{0}\right| .
$$

$$
-\Delta w_{1} \leq \frac{8}{9 \varepsilon^{2}}\left(1-w_{1}^{2}\right) w_{1}+\frac{4}{3 \mu \varepsilon}\left(1-w_{1}^{2}\right) .
$$

As previously, we may consider only $\mu \geq \frac{24 \varepsilon}{a}$, then $\frac{4}{3 \mu \varepsilon} \leq \frac{a}{18 \varepsilon^{2}} \leq \frac{w_{1}}{18 \varepsilon^{2}}$ and

$$
-\Delta w_{1} \leq\left(\frac{8}{9}+\frac{1}{18}\right) \frac{1}{\varepsilon^{2}} w_{1}\left(1-w_{1}^{2}\right) .
$$

We then define $w_{2}$ by $w_{2}=w_{1}-M \omega^{2} \varepsilon^{2}$. From (2.10), as $w_{2} \leq w_{1}$,

$$
\begin{aligned}
-\Delta w_{2}-\frac{1}{\varepsilon^{2}} w_{2}\left(1-w_{2}^{2}\right)+\omega^{2} r^{2} w_{2} & \leq \frac{17}{18 \varepsilon^{2}}\left(1-w_{1}^{2}\right) w_{1}-\frac{1}{\varepsilon^{2}} w_{2}\left(1-w_{2}^{2}\right)+\omega^{2} r^{2} w_{2} \\
& \leq \frac{17}{18 \varepsilon^{2}}\left(1-w_{2}^{2}\right)\left(w_{2}+M \omega^{2} \varepsilon^{2}\right)-\frac{1}{\varepsilon^{2}} w_{2}\left(1-w_{2}^{2}\right)+\omega^{2} r^{2} w_{2} \\
& \leq-\frac{1}{18 \varepsilon^{2}} w_{2}\left(1-w_{2}^{2}\right)+\frac{17}{18 \varepsilon^{2}}\left(1-w_{2}^{2}\right) M \omega^{2} \varepsilon^{2}+\omega^{2} r^{2} w_{2}
\end{aligned}
$$


But, for $\varepsilon$ small enough, $w_{2} \geq \frac{a}{2}$, hence

$$
\begin{aligned}
-\Delta w_{2}-\frac{1}{\varepsilon^{2}} w_{2}\left(1-w_{2}^{2}\right)+\omega^{2} r^{2} w_{2} & \leq-\frac{w_{2}}{\varepsilon^{2}}\left(\frac{1-w_{2}^{2}}{18}-\frac{17}{18}\left(1-w_{2}^{2}\right) \frac{2}{a} M \varepsilon^{2} \omega^{2}-\omega^{2} \varepsilon^{2} r^{2}\right) \\
& \leq-\frac{w_{2}}{\varepsilon^{2}}\left(\frac{1-w_{2}^{2}}{18}(1-o(1))-\omega^{2} \varepsilon^{2} r^{2}\right) .
\end{aligned}
$$

On the other hand,

for $\varepsilon$ small enough. Then,

$$
1-w_{2}^{2} \geq \frac{1}{2}\left(1-w_{2}\right) \geq \frac{M}{2} \omega^{2} \varepsilon^{2}
$$

$$
\frac{1-w_{2}^{2}}{18}(1-o(1))-\omega^{2} \varepsilon^{2} r^{2} \geq \frac{M}{40} \omega^{2} \varepsilon^{2}-r^{2} \omega^{2} \varepsilon^{2}>0
$$

if $M$ is chosen large enough compared to $\max _{\Omega} r$. Therefore, for a suitable choice of $M$,

$$
-\Delta w_{2}-\frac{1}{\varepsilon^{2}} w_{2}\left(1-w_{2}^{2}\right)+\omega^{2} r^{2} w_{2} \leq 0 \quad \text { on } B\left(x_{0}, \mu\right)
$$

and $w_{2} \leq a \leq \rho$ on $\partial B\left(x_{0}, \mu\right)$, hence $w_{2}$ is a subsolution for $(2.8)$ and we deduce that $\rho \geq w_{2} \geq w_{1}-M \omega^{2} \varepsilon^{2}$ on $B\left(x_{0}, \mu\right)$, and, as in [4],

$$
1-\rho \leq C \varepsilon^{-\frac{\mu}{2 \varepsilon}}+O\left(\omega^{2} \varepsilon^{2}\right) \quad \text { on } B\left(x_{0}, \mu\right) .
$$

As $\mu=\delta\left(x_{0}\right)-\frac{1}{2} K \varepsilon$, we obtain that

$$
1-\rho\left(x_{0}\right) \leq C \varepsilon^{-\frac{\delta\left(x_{0}\right)}{2 \varepsilon}}+O\left(\varepsilon^{2} \omega^{2}\right)
$$

and finally, changing $C$ if necessary, this estimate is true on all $\Omega$, which proves (2.5).

For (2.6), it is well-known (see [4] or [14]) that

$$
\min _{H_{0}^{1}(\Omega, \mathbb{R})} \frac{1}{2} \int_{\Omega}|\nabla u|^{2}+\frac{1}{2 \varepsilon^{2}}\left(1-u^{2}\right)^{2} \leq \frac{\sqrt{2}}{3 \varepsilon} l(\partial \Omega)+O(1) .
$$

Hence, by definition of $\rho$,

This implies that

$$
J(\rho) \leq \frac{\sqrt{2}}{3 \varepsilon} l(\partial \Omega)+\frac{\omega^{2}}{2} \int_{\Omega}|x|^{2}+O(1) .
$$

thus

$$
\frac{1}{4 \varepsilon^{2}} \int_{\Omega}\left(1-\rho^{2}\right)^{2}+\frac{\omega^{2}}{2} \int_{\Omega} r^{2} \rho^{2} \leq \frac{C}{\varepsilon}+\frac{\omega^{2}}{2} \int_{\Omega} r^{2}
$$

from which we deduce $(2.7)$.

$$
\frac{1}{4 \varepsilon^{2}} \int_{\Omega}\left(1-\rho^{2}\right)^{2} \leq \frac{C}{\varepsilon}+\frac{\omega^{2}}{2} \int_{\Omega} r^{2}\left(1-\rho^{2}\right) \leq \frac{C}{\varepsilon}+C \frac{\omega^{2}}{2}\left(\int_{\Omega}\left(1-\rho^{2}\right)^{2}\right)^{\frac{1}{2}},
$$

Thus, in the case of a disc domain, $\rho_{\varepsilon}$ is a vortex-less solution of (G.P.). As explained in the introduction, the fact that $u=0$ on $\partial \Omega$ induces a cost of $\frac{C}{\varepsilon}$ in the energy. That cost can be, as in [AS], removed by considering $v=\frac{u}{\rho}$. Then, $v \simeq u$ except near the boundary, and the boundary cost is "carried" by $\rho$. This can be proved by using the fact that the energy splits very conveniently under the decomposition $u=\rho v$, exactly as in [LM] or [AS]. More precisely, we have the following lemma, in which $H_{\rho^{2}}^{1}$ denotes the $H^{1}$ space with respect to the measure $\rho^{2} d x$, and the same for $L_{\rho^{2}}^{2}$. 
Lemma 2.2. Let $u \in H_{0}^{1}(\Omega, \mathbb{C}) . \exists \varepsilon_{0}, \forall \varepsilon<\varepsilon_{0}, v=\frac{u}{\rho}$ is well-defined, belongs to $H_{\rho^{2}}^{1}$ and

$$
J(u)=J(\rho)+\int_{\Omega} \frac{\rho^{2}}{2}|\nabla v|^{2}+\frac{\rho^{4}}{4 \varepsilon^{2}}\left(1-|v|^{2}\right)^{2}+\omega \int_{\Omega} \rho^{2}\left(i v, x_{2} v_{x_{1}}-x_{1} v_{x_{2}}\right) .
$$

Proof. Let $d x$ denote the Lebesgue measure on $\mathbb{R}^{2}$.

Step 1: We prove that $v \in H_{\rho^{2}}^{1}$.

$\rho$ only vanishes on $\partial \Omega$, hence $v=\frac{u}{\rho}$ is well-defined on $\Omega$. Furthermore,

$$
\int_{\Omega}|v|^{2} \rho^{2} d x=\int_{\Omega}|u|^{2} d x<\infty
$$

hence $v \in L_{\rho^{2}}^{2}$. Then, $\nabla v=\frac{\nabla u}{\rho}-\frac{u}{\rho^{2}} \nabla \rho$. As $u \in H_{0}^{1}(\Omega, \mathbb{C}), \frac{|\nabla u|^{2}}{\rho^{2}}$ is $\rho^{2} d x$ integrable. On the other hand, we can say from (2.5) that

$$
\exists \lambda>0, \quad \delta(x) \geq \lambda \varepsilon \Longrightarrow \rho_{\varepsilon}(x) \geq \frac{1}{2}
$$

Hence, we have

$$
\int_{\{x / \delta(x) \geq \lambda \varepsilon\}}\left|\frac{u}{\rho^{2}} \nabla \rho\right|^{2} \rho^{2} \leq C\|\nabla \rho\|_{L}^{2} \int_{\Omega}|u|^{2}<\infty
$$

while, with (2.5),

$$
\begin{aligned}
\int_{\{x / \delta(x) \leq \lambda \varepsilon\}}\left|\frac{u}{\rho^{2}} \nabla \rho\right|^{2} \rho^{2} & =\int_{\{x / \delta(x) \leq \lambda \varepsilon\}} \frac{|u|^{2}|\nabla \rho|^{2}}{\rho^{2}} \leq\|\nabla \rho\|_{L^{\infty}}^{2} \int_{\{x / \delta(x) \leq \lambda \varepsilon\}} C \varepsilon^{2} \frac{|u|^{2}}{\delta(x)^{2}} \\
& \leq C \varepsilon^{2}\|\nabla \rho\|_{L^{\infty}}^{2} \int_{\Omega}|\nabla u|^{2}<\infty
\end{aligned}
$$

where we have used the Hardy inequality.

Hence, we deduce that $\nabla v \in L_{\rho^{2}}^{2}$ and $v \in H_{\rho^{2}}^{1}$ with $\|v\|_{H_{\rho^{2}}^{1}} \leq C(\varepsilon)\|u\|_{H_{0}^{1}}$.

Step 2: We prove the splitting of the energy. This proof is very similar to that of [13] and [4], and was suggested by Shafrir. For any $t>0$, we denote $\Omega_{t}=\{x \in \Omega / \delta(x)>t\}$. For any $t>0$ sufficiently small, we have

$$
\begin{aligned}
\int_{\Omega_{t}} \frac{1}{2}|\nabla(\rho v)|^{2} & +\frac{1}{4 \varepsilon^{2}}\left(1-\rho^{2}|v|^{2}\right)^{2}+\frac{\omega^{2} r^{2}}{2} \rho^{2}|v|^{2}=\int_{\Omega_{t}} \frac{1}{2}|v|^{2}|\nabla \rho|^{2}+\frac{1}{4} \nabla \rho^{2} \cdot \nabla\left(|v|^{2}-1\right) \\
& +\frac{1}{2} \rho^{2}|\nabla v|^{2}+\int_{\Omega_{t}} \frac{1}{4 \varepsilon^{2}}\left(1-\rho^{2}+\rho^{2}\left(1-|v|^{2}\right)\right)^{2}+\frac{\omega^{2} r^{2}}{2} \rho^{2}+\frac{\omega^{2} r^{2}}{2} \rho^{2}\left(|v|^{2}-1\right) \\
= & \int_{\Omega_{t}} \frac{1}{2}|\nabla \rho|^{2}+\frac{1}{4 \varepsilon^{2}}\left(1-\rho^{2}\right)^{2}+\frac{\omega^{2} r^{2}}{2} \rho^{2}++\frac{1}{4 \varepsilon^{2}} \rho^{4}\left(1-|v|^{2}\right)^{2}+\frac{1}{2} \rho^{2}|\nabla v|^{2} \\
& +\int_{\Omega_{t}}\left(|v|^{2}-1\right)\left(-\frac{1}{4} \Delta\left(\rho^{2}\right)+\frac{\omega^{2} r^{2}}{2} \rho^{2}-\frac{1}{2 \varepsilon^{2}} \rho^{2}\left(1-\rho^{2}\right)+\frac{1}{2}|\nabla \rho|^{2}\right) \\
& +\frac{1}{2} \int_{\partial \Omega_{t}} \rho \frac{\partial \rho}{\partial n}\left(|v|^{2}-1\right) .
\end{aligned}
$$


Now, since $\rho$ satisfies (2.4), we have

$$
-\Delta \rho^{2}=2 \rho(-\Delta \rho)-2|\nabla \rho|^{2}=-2 \omega^{2} r^{2} \rho^{2}+\frac{2}{\varepsilon^{2}}\left(1-\rho^{2}\right) \rho^{2}-2|\nabla \rho|^{2},
$$

so that

$$
\begin{aligned}
\int_{\Omega_{t}} \frac{1}{2}|\nabla u|^{2}+\frac{1}{4 \varepsilon^{2}}\left(1-|u|^{2}\right)^{2}+\frac{\omega^{2} r^{2}}{2}|u|^{2}= & \int_{\Omega_{t}} \frac{1}{2}|\nabla \rho|^{2}+\frac{1}{4 \varepsilon^{2}}\left(1-\rho^{2}\right)^{2} \\
& +\frac{\omega^{2} r^{2}}{2} \rho^{2}+\int_{\Omega_{t}} \frac{1}{2} \rho^{2}|\nabla v|^{2}+\frac{\rho^{4}}{4 \varepsilon^{2}}\left(1-|v|^{2}\right)^{2} \\
& +\frac{1}{2} \int_{\partial \Omega_{t}} \rho \frac{\partial \rho}{\partial n}\left(|v|^{2}-1\right) .
\end{aligned}
$$

But, from the properties stated on $\rho$, we have

$$
\left.\left|\int_{\partial \Omega_{t}} \rho \frac{\partial \rho}{\partial n}\right| v\right|^{2}\left|\leq \int_{\partial \Omega_{t}}\right| \frac{\partial \rho}{\partial n} \mid \frac{|u|^{2}}{\rho} \leq C \varepsilon\|\nabla \rho\|_{L^{\infty}} \int_{\partial \Omega_{t}} \frac{|u|^{2}}{\delta(x)} .
$$

From the Hardy inequality,

$$
\int_{\Omega} \frac{|u|^{2}}{\delta(x)^{2}} \leq C \int_{\Omega}|\nabla u|^{2}<\infty
$$

hence, we can find a sequence $t_{n} \rightarrow 0$ such that

$$
\int_{\partial \Omega_{t_{n}}} \frac{|u|^{2}}{\delta(x)^{2}} \leq \frac{C}{t_{n}\left|\log t_{n}\right|}
$$

therefore,

$$
\int_{\partial \Omega_{t_{n}}} \frac{|u|^{2}}{\delta(x)}=\int_{\partial \Omega_{t_{n}}} \frac{|u|^{2}}{t_{n}} \leq \frac{C}{\left|\log t_{n}\right|} \longrightarrow 0 \quad \text { as } n \rightarrow \infty
$$

On the other hand,

$$
\lim _{t \rightarrow 0} \int_{\partial \Omega_{t}} \rho \frac{\partial \rho}{\partial n}=0
$$

Applying (2.15) to $t=t_{n}$, and passing to the limit $n \rightarrow \infty$, we obtain

$$
\int_{\Omega} \frac{1}{2}|\nabla u|^{2}+\frac{1}{4 \varepsilon^{2}}\left(1-|u|^{2}\right)^{2}+\frac{\omega^{2} r^{2}}{2}|u|^{2}=J(\rho)+\int_{\Omega} \frac{1}{2} \rho^{2}|\nabla v|^{2}+\frac{\rho^{4}}{4 \varepsilon^{2}}\left(1-|v|^{2}\right)^{2} .
$$

There remains to deal with

$$
\int_{\Omega}\left(i u, x_{2} u_{x_{1}}-x_{1} u_{x_{2}}\right) .
$$

But replacing $u$ by $\rho v$, we obtain that this term is equal to

$$
\int_{\Omega} \rho^{2}\left(i v, x_{2} v_{x_{1}}-x_{1} v_{x_{2}}\right)+\int_{\Omega} \rho(i v, v)\left(x_{2} \rho_{x_{1}}-x_{1} \rho_{x_{2}}\right),
$$

where the second term vanishes identically. Hence we have the desired result. 


\section{Branches of vortex solutions in the CASE of THE DisC}

In this section $\Omega=B(0, R)=B_{R}$. We consider rotation speeds

$$
\omega \leq C \varepsilon^{-\alpha}
$$

for $\alpha$ sufficiently small, to be specified in the proof, and obtain similar results as those of [16-18] concerning branches of solutions of (G.P.).

As mentioned in the introduction, we cannot study zeros of $u$ close to $\partial \Omega$ because $|u|$ vanishes at the boundary and is smaller than $\frac{1}{2}$ on a layer of size $\lambda \varepsilon$ near the boundary. On the opposite, we can study vortices of $v$, which does not have to vanish on $\partial \Omega$, and vortices of $v$ are vortices of $u$. But it is difficult to get information on $v$ and its vortices near the boundary, and anyway, it is not very relevant, since $u$ has something like a layer of vortices of size $\varepsilon$ near the boundary. This is why we restrict to studying $v$ on the domain $\left\{x \in \Omega, \delta(x) \geq \varepsilon^{\beta}\right\}$ where $\beta$ is some constant $<1$ and close to 1 . Furthermore, there are no boundary conditions on $v$, hence we can adjust the techniques of [16-18] to $v$. The rest of this section is just these adjustments.

\subsection{Defining the domains of minimization}

We perform, as in [16-18], local minimizations of $J$ over well-chosen domains.

First, Lemma 2.2 has allowed us to separate the very strongly-divergent part $J\left(\rho_{\varepsilon}\right)$ (in $\frac{C}{\varepsilon}$ ) from the rest which is very similar to the Ginzburg-Landau energy functional $J$ of [16-18], and diverges at most in $C \omega^{2}$. We use here the notation of [16]:

$$
F(v)=\frac{1}{2} \int_{\Omega}|\nabla v|^{2}+\frac{1}{2 \varepsilon^{2}}\left(1-|v|^{2}\right)^{2}
$$

for the energy functional studied in [5]. We shall also write $F_{V}$ for the functional $F$ restricted to any subdomain $V$ of $\Omega$.

Then, we denote

$$
G(v)=\frac{1}{2} \int_{\Omega} \rho^{2}|\nabla v|^{2}+\frac{\rho^{4}}{2 \varepsilon^{2}}\left(1-|v|^{2}\right)^{2},
$$

for the "weighted" BBH-functional that appears naturally in the splitting of $J$.

We then define the following mappings:

$$
\begin{aligned}
& H^{1} \longrightarrow H_{\rho^{2}}^{1} \\
& u \longmapsto v=\frac{u}{\rho}
\end{aligned}
$$

is a continuous mapping, as proved in Section 2. So is

$$
\begin{aligned}
& H_{\rho^{2}}^{1} \longrightarrow H_{\rho^{2}}^{1} \\
& v \longmapsto T(v)=\mid \begin{array}{l}
\frac{v}{\rho|v|} \text { if }|v| \geq \frac{1}{\rho} \\
v \text { otherwise. }
\end{array}
\end{aligned}
$$

In addition, we have the following lemma, whose proof is postponed to the end of this section:

Lemma 3.1. 1) For any $u \in H_{0}^{1}(\Omega, \mathbb{C})$,

$$
|T(v)| \leq \frac{1}{\rho}
$$


2) There exists $\alpha>0$ such that, for any $u$ satisfying $J(u) \leq J(\rho)$, if $\omega \leq C \varepsilon^{-\alpha}$,

$$
J(\rho T(v)) \leq J(\rho v)+o(1) \quad \text { as } \varepsilon \rightarrow 0 .
$$

This lemma means that, if we replace $u$ by $\rho T(v)$, we get a function with values in $\overline{B(0,1)}$ and with a lower energy than $u$, up to a small error term. Hence, we can make this replacement to find local minimizers.

We now define the domains of minimization. A real (large) positive constant $\mathcal{M} \notin \pi \mathbb{N} \quad(\mathcal{M}>\pi)$ being set, we define (as in [16]),

$$
D_{\mathcal{M}}=\left\{u \in H_{0}^{1}\left(B_{R}, \mathbb{C}\right) / G \circ T\left(\frac{u}{\rho}\right)<\mathcal{M}|\log \varepsilon|\right\}
$$

This is going to be our largest domain of minimization. We shall also use smaller domains of minimization, of the form

$$
D_{a, b}=\left\{u \in H_{0}^{1}\left(B_{R}, \mathbb{C}\right) / a<\frac{G \circ T\left(\frac{u}{\rho}\right)}{|\log \varepsilon|}<b\right\},
$$

where $a, b<\mathcal{M}$ but may depend on $\varepsilon$. $D_{\mathcal{M}}$ and $D_{a, b}$ are open domains in $H_{0}^{1}$, from the continuity of the mappings (3.3) and (3.4).

\subsection{Definition of the regularized map and its vortices}

We wish to study minimizers of $J$ in $D_{\mathcal{M}}$ or $D_{a, b}$. Considering any $u$ in one of these domains, we write $v=T\left(\frac{u}{\rho}\right)$, so that $|v| \leq \frac{1}{\rho}$ and, thanks to Lemma 3.1, we can study $v$ instead of $\frac{u}{\rho}$.

As in [16-18], we need to define vortices of $v$ for any $u \in D_{\mathcal{M}}$. But, exactly as in these papers, this is impossible to do directly because $u$ is not a priori solution of (G.P.) hence there is no upper bound of the type $|\nabla u| \leq \frac{C}{\varepsilon}$ on its gradient. As in [16-18], in order to define vortices of $v$, we replace it, following the method of [1], by a regularized map $v^{\gamma}$ which has well-defined vortices. First, we remove the boundary of the domain, by setting

$$
B^{\prime}=B_{R} \backslash\left\{x / \delta(x) \leq \varepsilon^{\beta}\right\},
$$

where $\beta$ is some constant $\in] 0,1[$. From (2.5),

$$
0 \leq 1-\rho \leq C \varepsilon^{1-\beta}+O\left(\varepsilon^{2} \omega^{2}\right)=o(1) \text { in } B^{\prime},
$$

hence we can consider $\rho$ as being equal to 1 in $B^{\prime}$. Then, our regularized map $v^{\gamma}$ is defined from $v$ to be the solution of the following problem:

$$
\min _{H^{1}\left(B^{\prime}, \mathbb{C}\right)} \int_{B^{\prime}} \frac{1}{2}|\nabla w|^{2}+\frac{\left(1-|w|^{2}\right)^{2}}{4 \varepsilon^{2}}+\frac{|v-w|^{2}}{2 \varepsilon^{2 \gamma}},
$$

where $\gamma$ is some constant in $] 0,1[$.

Exactly as in [16-18] and [1], this $v^{\gamma}$ has the same behaviour as $v$ at scales larger than $\varepsilon^{\gamma}$ (it is a parabolic regularization of $v$ ), hence its vortex-structure and behaviour with respect to $J$ are going to be almost the same as those of $v$, as we shall prove.

Lemma 3.2. If $u \in D_{\mathcal{M}}$ and $v=T\left(\frac{u}{\rho}\right)$, then $v^{\gamma}$ satisfies

$$
\left|\nabla v^{\gamma}\right| \leq \frac{C}{\varepsilon} \quad\left|v^{\gamma}\right| \leq \frac{1}{\rho}
$$




$$
F_{B^{\prime}}\left(v^{\gamma}\right) \leq F_{B^{\prime}}(v) \leq G(v)+o(1)
$$

Proof. The first assertions are standard (recall that $|v| \leq \frac{1}{\rho}$ ). For the second result, take $v$ as a test-map in (3.10). Then, observe that

$$
\begin{aligned}
F_{B^{\prime}}(v)-G(v) & \leq \int_{B^{\prime}} \frac{1}{2}\left(1-\rho^{2}\right)|\nabla v|^{2}+\frac{\left(1-\rho^{4}\right)}{4 \varepsilon^{2}}\left(1-|v|^{2}\right)^{2} \leq C\|1-\rho\|_{L^{\infty}\left(B^{\prime}\right)} G(v) \\
& \leq C\left(\varepsilon^{1-\beta}+\varepsilon^{1-\alpha}\right)|\log \varepsilon|=o(1)
\end{aligned}
$$

where we have used (3.9) and the fact that $u \in D_{\mathcal{M}}$.

Then, as in [16], Proposition 3.2, we deduce from the analysis of [1], that we can define vortices of size $\sigma$ of $v^{\gamma}$ satisfying the following properties:

Lemma 3.3. Let $0<\gamma<\beta<\mu<1$, with $\bar{\mu}=\mu^{N+1}>\beta$; for $\varepsilon$ sufficiently small, we may find a bounded number of balls $\left(B\left(a_{i}, \sigma\right)\right)_{i \in \mathcal{J}}$ such that the following properties hold

$$
\begin{aligned}
& \lambda \varepsilon \leq \varepsilon^{\mu} \leq \sigma \leq \varepsilon^{\bar{\mu}}<\varepsilon^{\beta}, \\
& \left|v^{\gamma}(x)\right| \geq \frac{1}{2} \quad \text { if } x \in B^{\prime} \backslash \bigcup_{i \in \mathcal{J}} B\left(a_{i}, \sigma\right), \\
& \left|v^{\gamma}(x)\right| \geq 1-\frac{2}{|\log \varepsilon|^{2}} \quad \text { if } x \in \partial B\left(a_{i}, \sigma\right), \quad \text { for } i \in \mathcal{J}, \\
& \int_{\partial B\left(a_{i}, \sigma\right)} e_{\varepsilon}\left(v^{\gamma}\right) \leq \frac{C(\beta, \mu)}{\sigma} \quad \text { for } i \in \mathcal{J}, \\
& \left|a_{i}-a_{j}\right| \geq 8 \sigma \quad \text { for any } i \neq j \in \mathcal{J} .
\end{aligned}
$$

We shall also write

$$
d_{i}=\operatorname{deg}\left(v^{\gamma}, \partial B\left(a_{i}, \sigma\right)\right) \neq 0
$$

$u$ or $v$ will be called "vortex-less" if $\mathcal{J}=\varnothing$ i.e. if $\left|v^{\gamma}\right| \geq \frac{1}{2}$ in $B^{\prime}$. It is proved in [16] Proposition 6.2, that this implies (if $J(u) \leq J(\rho)$ ) that $|v| \geq \frac{1}{2}$ in $B^{\prime}$.

Similarly, if $v$ itself has well-defined vortices, then there is a close link between them and those of $v^{\gamma}$, see [16], Proposition 3.3.

From now on, we denote $\left(\left(a_{i}, d_{i}\right)\right)_{i=1}^{l}$ the vortices of $v^{\gamma}$, also called "vortices of $u$ " or "vortices of $v$ " by extension. We have the following lower bound, borrowed from [16], Proposition 5.1 and Lemma 5.1:

\section{Proposition 3.1.}

$$
\begin{gathered}
F_{B^{\prime}}\left(v^{\gamma}\right) \geq \pi \sum_{i \in \mathcal{J}}\left|d_{i}\right| \log \frac{\sigma}{\varepsilon}+O(1) \\
F_{B^{\prime}}\left(v^{\gamma}\right) \geq \pi \sum_{i \in \mathcal{J}} d_{i}^{2}|\log \sigma|+\pi \sum_{i \in \mathcal{J}}\left|d_{i}\right| \log \frac{\sigma}{\varepsilon}+W\left(\left(a_{1}, d_{1}\right), \cdots,\left(a_{l}, d_{l}\right)\right)+O(1),
\end{gathered}
$$

where

$$
W=-\pi \sum_{i \neq j} d_{i} d_{j} \log \left|a_{i}-a_{j}\right|-\pi \sum_{i=1}^{l} d_{i} R_{0}\left(a_{i}\right)
$$

and $R_{0}$ is the solution of

$$
\begin{cases}\Delta R_{0}=0 & \text { in } \Omega \\ R_{0}=-\pi \sum_{i=1}^{l} d_{i} \log \left|x-a_{i}\right| & \text { on } \partial \Omega\end{cases}
$$




\subsection{Expanding the energy-functional}

Having a definition of vortices, we use it to expand the energy functional and get an expansion which is totally similar to that of $[16]$.

First, we have the following lemma, the proof of which is left to the end of the section.

Lemma 3.4. If $\omega \leq C \varepsilon^{-\alpha}$, for some $\alpha>0$ sufficiently small, we have

$$
\begin{aligned}
\omega \int_{B_{R}}\left(i u, x_{2} u_{x_{1}}-x_{1} u_{x_{2}}\right) & =\omega \int_{B_{R}} \rho^{2}\left(i v, x_{2} v_{x_{1}}-x_{1} v_{x_{2}}\right)=\omega \int_{B_{R}} \rho^{2}(i v, d v \wedge d X) \\
& =2 \pi \omega \sum_{i \in \mathcal{J}} d_{i} X\left(a_{i}\right)+o(1) \quad \text { as } \varepsilon \rightarrow 0
\end{aligned}
$$

where $X$ is the function defined on $B_{R}$ as

$$
X(x)=\frac{|x|^{2}-\left(R-\varepsilon^{\beta}\right)^{2}}{2},
$$

so that

$$
X=0 \quad \text { on } \partial B^{\prime}
$$

Once this lemma is proved, the expansion of the energy follows very easily from (2.12):

Proposition 3.2. There exists $\alpha>0$ such that, if $\omega \leq C \varepsilon^{-\alpha}, u \in D_{\mathcal{M}}$ and $|u| \leq 1$,

$$
J(u)=J(\rho)+G(v)+2 \pi \omega \sum_{i \in \mathcal{J}} d_{i} X\left(a_{i}\right)+o(1) \quad \text { as } \varepsilon \rightarrow 0,
$$

where $G$ was defined in (3.2).

It is easy to deduce from this proposition, as in [16], that the minimal energy over vortex-less configurations is $J(\rho)+o(1)$ as $\varepsilon \rightarrow 0$. Actually, it is smaller than $J(\rho)$, and it should be $J(\rho)$ since $\rho$ is a solution of (G.P.). One could hope to prove, following the method of [17], the uniqueness of a vortex-less solution, hence the minimality of $\rho$ among them, but the problem is that the notion of "vortex-less" is difficult to define up to the boundary of the domain. Hence it remains an open problem to know whether $\rho$ is minimal for $\omega \leq \omega_{1}$.

\subsection{The critical velocity}

We are now in a position to deduce the critical velocity, that we denote $\omega_{1}$, by analogy with the first critical field $H_{c_{1}}$ for superconductors in [16].

$\omega_{1}$ is defined to be the largest value of $\omega$ below which the minimum of $J$ is larger than $J(\rho)-o(1)$. For $\omega \geq \omega_{1}$, minimizers of $J$ will have vortices. We will look for them in $D_{\mathcal{M}}$, domain which corresponds roughly (in view of Prop. 3.1) to configurations with less than $\frac{\mathcal{M}}{\pi}$ vortices.

We get the following theorem, which is completely analogous to Theorem 1 of [16]:

Theorem 1. Defining a rotation velocity $\omega_{1}$ by

$$
\omega_{1}=\frac{|\log \varepsilon|}{R^{2}}
$$

there exist $k(\varepsilon)=O(1), k^{\prime}(\varepsilon)=O(|\log | \log \varepsilon||)$, and $\varepsilon_{0}(\mathcal{M})$ such that for $\varepsilon<\varepsilon_{0}$, the following holds:

- if and only if $\omega \leq \omega_{1}-k(\varepsilon)$, the minimum of $J$ is $J(\rho)-o(1)$ and if $\omega \leq \omega_{1}-k^{\prime}(\varepsilon)$ any minimizer satisfies $|v| \geq \frac{1}{2}$ on $B^{\prime} \cdot\left(v=T\left(\frac{u}{\rho}\right)\right)$ 
- if $\omega_{1}+k(\varepsilon) \leq \omega \leq \omega_{1}+O(1)$, there exists a minimizer of $J$ over $D_{\mathcal{M}}$ which is a solution of (G.P.). In addition, "it" has exactly one vortex a of degree one, and $|a| \rightarrow 0$ as $\varepsilon \rightarrow 0$.

Proof.

Step 1: Upper bound for the minimal energy.

We start from Proposition 3.2 and

$$
J(u)=J(\rho)+G(v)+2 \pi \omega \sum_{i \in \mathcal{J}} d_{i} X\left(a_{i}\right)+o(1) .
$$

We can construct a configuration the following way: we choose $v$ such that $|v| \leq 1, v$ has a zero of degree one at the center of $B_{R}$, and

$$
F(v)=\frac{1}{2} \int_{B_{R}}|\nabla v|^{2}+\frac{1}{2 \varepsilon^{2}}\left(1-|v|^{2}\right)^{2} \leq \pi|\log \varepsilon|+O(1) .
$$

This is possible exactly as in [16], proof of Proposition 6.1. Then, defining $u=\rho v$, it is easy to check that $u \in D_{\mathcal{M}}$ and

$$
\begin{aligned}
& J(u) \leq J(\rho)+F(v)+2 \pi \omega X(0)+o(1) \\
& J(u) \leq J(\rho)+\pi|\log \varepsilon|-\pi \omega R^{2}+O(1)
\end{aligned}
$$

in view of the definition (3.13).

Hence, we have

$$
\min _{D_{\mathcal{M}}} J \leq \min \left(J(\rho), J(\rho)+\pi|\log \varepsilon|-\pi \omega R^{2}+O(1)\right),
$$

and

$$
\min _{D_{\mathcal{M}}} J<J(\rho)-1
$$

if

$$
\frac{|\log \varepsilon|}{R^{2}}+k(\varepsilon) \leq \omega \leq C \varepsilon^{-\alpha}
$$

for some $k(\varepsilon)=O(1)$.

$\min _{D_{\mathcal{M}}} J<J(\rho)$ for $\omega \geq C \varepsilon^{-\alpha}$ will be proved in the next section.

Step 2: Study of the minimizers of $J$ around $\omega_{1}$.

Let $u$ achieving min $J$ over $\overline{D_{\mathcal{M}}}$ (such a $u$ exists), and let $v=T\left(\frac{u}{\rho}\right), v^{\gamma}$ its regularized map. From Lemma 3.1, we have

$$
J(\rho v) \leq \frac{\min }{D_{\mathcal{M}}} J+o(1) \leq J(\rho)+o(1) .
$$

On the other hand, thanks to Proposition 3.2, and (3.11),

$$
\begin{aligned}
J(u) & =J(\rho)+G(v)+2 \pi \omega \sum_{i} d_{i} X\left(a_{i}\right)+o(1) \\
& \geq J(\rho)+F_{B^{\prime}}\left(v^{\gamma}\right)+2 \pi \omega \sum_{i} d_{i} X\left(a_{i}\right)+o(1) .
\end{aligned}
$$


For $\omega \leq \omega_{1}+O(1)$, we have by minimality of $u$ in $\overline{D_{\mathcal{M}}}$,

$$
J(u) \leq \frac{\min }{D_{\mathcal{M}}} J \leq \min \left(J(\rho), J(\rho)+\pi|\log \varepsilon|-\pi \omega R^{2}+O(1)\right)
$$

hence

$$
F_{B^{\prime}}\left(v^{\gamma}\right)+2 \pi \omega \sum_{i} d_{i} X\left(a_{i}\right) \leq \min \left(o(1), \pi|\log \varepsilon|-\pi \omega R^{2}+O(1)\right) \leq O(1) .
$$

Comparing this to Proposition 3.1, which asserts that

$$
F_{B^{\prime}}\left(v^{\gamma}\right) \geq \pi \sum_{i}\left|d_{i}\right| \log \frac{\sigma}{\varepsilon}+O(1) \geq \pi(1-\mu) \sum_{i}\left|d_{i}\right||\log \varepsilon|+O(1),
$$

we deduce (exactly as in [16], proof of Lem. 6.1), that

$$
d_{i}>0 \quad \forall i \in \mathcal{J},
$$

because $\min X=X(0)=-\frac{R^{2}}{2}+o(1)$.

In view of the expression of $W$, exactly as in [16], this implies that $W \geq O(1)$, and that the following lower bounds hold:

$$
\begin{aligned}
& F_{B^{\prime}}\left(v^{\gamma}\right) \geq \pi\left(\sum_{i}\left|d_{i}\right|^{2} \log \frac{1}{\sigma}+\sum_{i}\left|d_{i}\right| \log \frac{\sigma}{\varepsilon}\right)+O(1) \\
& F_{B^{\prime}}\left(v^{\gamma}\right) \geq \pi \sum_{i}\left|d_{i}\right| \log \frac{1}{\varepsilon}+O(1) .
\end{aligned}
$$

For some $k(\varepsilon)=O(1)$, if $\omega \leq \omega_{1}+k(\varepsilon)$, combining this with (3.20) and (3.21) implies that $\mathcal{J}=\varnothing$, hence that $\left|v^{\gamma}\right| \geq \frac{1}{2}$ on $B^{\prime}$. From the analysis of [16], (see Prop. 6.2), this allows to prove that $|v| \geq \frac{1}{2}$ on $B^{\prime}$, i.e. that $v$ is vortex-less on $B^{\prime}$.

On the other hand, if $\omega \geq \omega_{1}+O(1)$, the comparison of (3.24) and (3.22) yields $d_{i}=1, \forall i \in \mathcal{J}$, and $X\left(a_{i}\right) \rightarrow \min X$, hence $v^{\gamma}$ only has vortices of degree 1 , tending to the origin. But then, if Card $\mathcal{J}>1$, i.e. if $v^{\gamma}$ has more than one vortex, its vortices repell one another because $W \rightarrow+\infty$ as $\left|a_{i}-a_{j}\right| \rightarrow 0$, and we are in this case since all the vortices tend to 0. Then, using Proposition 3.1,

$$
F_{B^{\prime}}\left(v^{\gamma}\right) \geq \pi \sum_{i}\left|d_{i}\right| \log \frac{1}{\varepsilon}+W+O(1)
$$

where $W \rightarrow+\infty$ if Card $\mathcal{J} \geq 2$. This is in contradiction with (3.20) and (3.18) if $\omega \leq \omega_{1}+O(1)$. Hence, for $\omega \leq \omega_{1}+O(1)$, any minimizer in $\overline{D_{\mathcal{M}}}$ has exactly one vortex of degree one tending to 0 .

Step 3: We can easily adjust the method of [20] to prove that, for $\omega \leq \omega_{1}-k^{\prime}(\varepsilon)$, a global minimizer of $J$ is vortex-less, and belongs to $D_{\mathcal{M}}$.

Step 4: There remains to prove that a minimizer of $J$ in $\overline{D_{\mathcal{M}}}$ is a solution of (G.P.) if $\frac{\mathcal{M}}{\pi} \notin \mathbb{N}$. This can be done exactly as in the forthcoming proof of Lemma 3.6, therefore we omit the details.

\subsection{Branches of $n$-vortices solutions}

In this section, we adjust the results of [18] concerning the existence of branches of stable solutions with $n$ vortices, for any $n<\frac{\mathcal{M}}{\pi}$. 
For any $n \geq 1$, we consider the domain

$$
U_{n}=\left\{u \in H_{0}^{1}\left(B_{R}, \mathbb{C}\right) / n|\log \varepsilon|+K<G\left(T\left(\frac{u}{\rho}\right)\right)<\left(n+\frac{1}{2}\right)|\log \varepsilon|\right\},
$$

where $K$ is a constant, to be set later. This domain is some $D_{a, b}$, but for simplicity, we denote it $U_{n}$ as in [18]. We begin with two lemmas:

Lemma 3.5. Suppose $\omega$ is any function of $\varepsilon$ tending to $+\infty$ as $\varepsilon \rightarrow 0$, and such that $\omega \leq C \varepsilon^{-\alpha}$. We have

$$
\frac{\inf }{U_{n}} J=J(\rho)+\pi n\left(|\log \varepsilon|-R^{2} \omega\right)+\frac{\pi}{2}\left(n^{2}-n\right) \log \omega+w\left(\tilde{a_{1}}, \cdots, \tilde{a_{n}}\right)+Q_{n}+o(1)
$$

where $\tilde{a}_{i}=a_{i} \sqrt{\omega}$, the $a_{i}$ 's being the vortices of a minimizer, $w$ is defined as

$$
w\left(x_{1}, \cdots, x_{n}\right)=-\pi \sum_{i \neq j} \log \left|x_{i}-x_{j}\right|+\pi \sum_{i}\left|x_{i}\right|^{2},
$$

and $Q_{n}$ is a constant depending only on $n$.

Proof. This lemma is exactly similar to Lemma 3.1 of [18]. We only give the main ideas of the proof. (3.27) relies on the expansion of Proposition 3.2, from which we have obtained the lower bound (3.20):

$$
J(u) \geq J(\rho)+F_{B^{\prime}}\left(v^{\gamma}\right)+2 \pi \omega \sum_{i} d_{i} X\left(a_{i}\right)+o(1)
$$

Then, $F_{B^{\prime}}$ is bounded from below exactly as in [16] using Proposition 3.1, and $W$ is expanded.

Actually, we construct a test configuration as in the proof of Theorem 1, for which the equality holds in (3.27) and that has $n$ vortices of degree 1 , located on a regular polygon of edge-size $\frac{1}{\sqrt{\omega}}$, centered at 0 . Using the explicit expression of $X$ and expanding $W$, we get the expression (3.27) as an upper bound for inf $\overline{U_{n}} J$.

For the lower bound, we begin by noticing that the upper bound implies $d_{i}=1, \forall i \in \mathcal{J}$, and $a_{i} \rightarrow 0$ as for Theorem 1, for the vortices of a minimizing map in $\overline{U_{n}}$. Then, the lower bound is obtained from (3.29), expanding $X$ and $W$ as previously, and performing the rescaling $\tilde{a_{i}}=a_{i} \sqrt{\omega}$ on the vortices.

Lemma 3.6. If $\varepsilon$ is sufficiently small, $\omega \rightarrow+\infty$ and $\omega \leq C \varepsilon^{-\alpha}$, $\min _{\overline{U_{n}}} J$ is achieved, and it is not achieved on $\partial U_{n}$.

Proof. It is exactly the same as the proof of Lemma 3.2 in [18]. We consider $u_{k}$ a minimizing sequence in $U_{n}$ and $v_{k}=T\left(\frac{u_{k}}{\rho}\right)$. We regularize them into $v_{k}^{\gamma}$ with vortices $\left(a_{i}(k), d_{i}(k)\right)$. Then, thanks to Proposition 3.2,

$$
J\left(\rho v_{k}\right)=J(\rho)+G(v)+2 \pi \omega \sum_{i} d_{i}(k) X\left(a_{i}(k)\right)+o_{\varepsilon}(1) \leq \inf _{U_{n}} J+o_{\varepsilon}(1)+o_{k}(1)
$$

Then,

$$
\inf _{\overline{U_{n}}} J \geq J(\rho)+F_{B^{\prime}}\left(v_{k}^{\gamma}\right)+\pi \omega \sum_{i} d_{i}(k)\left(\left|a_{i}(k)\right|^{2}-R^{2}\right)+o_{\varepsilon}(1)+o_{k}(1) .
$$

Comparing this with the expression (3.27) for $\inf _{\overline{U_{n}}} J$, we deduce that, for small $\varepsilon$, all $d_{i}(k)$ are positive, that $\sum_{i}\left|d_{i}(k)\right|=n$ (we already knew that $\sum_{i}\left|d_{i}(k)\right| \leq n$ since $u_{k} \in \overline{U_{n}}$ ), and that $\sum_{i}\left|a_{i}(k)\right|^{2} \rightarrow 0$ as $\varepsilon \rightarrow 0$.

Up to the extraction of a subsequence, $u_{k} \rightarrow u$ in $H_{0}^{1}$ and, by lower semi-continuity, $J(u) \leq \inf _{\overline{U_{n}}} J$. 
There remains to prove that $u \in \overline{U_{n}}$. This can be proved as in [18], using the idea that the weak $H^{1}$ convergence preserves the vortices. One proves that, if $v=T\left(\frac{u}{\rho}\right)$, and $\left(b_{i}, q_{i}\right)$ are the vortices of $v^{\gamma}$, then

$$
\pi \sum_{i} d_{i}(k)\left(\left|a_{i}(k)\right|-R^{2}\right) \simeq \int_{B^{\prime}}\left(i v_{k}, d v_{k} \wedge d X\right) \underset{k \rightarrow \infty}{\longrightarrow} \int_{B^{\prime}}(i v, d v \wedge d X) \simeq \pi \sum_{i} q_{i}\left(\left|b_{i}\right|^{2}-R^{2}\right) .
$$

On the one hand, we have, by definition of $U_{n}$,

$$
G(v) \leq \liminf _{k \rightarrow+\infty} G\left(T\left(\frac{u_{k}}{\rho}\right)\right) \leq \pi\left(n+\frac{1}{2}\right)|\log \varepsilon|,
$$

leading to $\sum_{i}\left|q_{i}\right| \leq n$. On the other hand (3.31) and the previous results on $\left(a_{i}(k), d_{i}(k)\right)$ yield $\sum_{q_{i}>0} q_{i} \geq n$, hence $\sum_{i}\left|q_{i}\right|=n$ and

$$
G(v) \geq F_{B^{\prime}}(v)-o(1) \geq F_{B^{\prime}}\left(v^{\gamma}\right)-o(1) \geq \pi n|\log \varepsilon|+O(1) .
$$

This proves, if $K$ is chosen small enough, that $u \in \overline{U_{n}}$ for small $\varepsilon$. Therefore, $\inf _{\overline{U_{n}}} J$ is achieved.

Step 2: We prove that the minimum is not achieved on $\partial U_{n}$. We follow the proof of Theorem 2 in [18]. By contradiction, suppose it is, then there is a minimizer $u$ such that

$$
G\left(T\left(\frac{u}{\rho}\right)\right)=\pi\left(n+\frac{1}{2}\right)|\log \varepsilon|
$$

or

$$
G\left(T\left(\frac{u}{\rho}\right)\right)=\pi n|\log \varepsilon|+K
$$

If (3.32) holds, then, with the usual notations, $\sum_{i}\left|d_{i}\right| \leq n$ and

$$
\begin{aligned}
\frac{\inf }{U_{n}} J & =J(\rho)+G\left(T\left(\frac{u}{\rho}\right)\right)+2 \pi \omega \sum_{i} d_{i} X\left(a_{i}\right)+o(1) \\
& \geq J(\rho)+\pi\left(n+\frac{1}{2}\right)|\log \varepsilon|-\pi \omega \sum_{i}\left|d_{i}\right| R^{2}+o(1) \\
& \geq J(\rho)+\pi n\left(|\log \varepsilon|-\omega R^{2}\right)+\frac{\pi}{2}|\log \varepsilon|+o(1)
\end{aligned}
$$

Comparing this to (3.27), we are led to

$$
\frac{\pi}{2}\left(n^{2}-n\right) \log \omega+O(1) \geq \frac{\pi}{2}|\log \varepsilon|,
$$

which is a contradiction as soon as $\omega \leq C \varepsilon^{-\alpha}$ for $\alpha$ sufficiently small compared to $\mathcal{M}$. The second possibility is (3.33). From the proof of the first step of the lemma, we know that, since $u$ minimizes $J$ in $\overline{U_{n}}$, we must have $\sum_{i}\left|d_{i}\right|=n\left(\right.$ and $\left.\sum_{i}\left|a_{i}\right|^{2} \rightarrow 0\right)$. But then,

$$
F_{B^{\prime}}(v) \geq \pi n|\log \varepsilon|+O(1)>\pi n|\log \varepsilon|+K,
$$

if $K$ is chosen small enough. Hence we get that (3.33) is impossible, and this concludes the proof.

We are now able to derive from these lemmas the following theorem, analogous to Theorem 2 in [17]. 
Theorem 2. Suppose $\omega$ is any function of $\varepsilon$ such that $\omega \rightarrow+\infty$ as $\varepsilon \rightarrow 0$, and $\omega \leq C \varepsilon^{-\alpha}$, then $\forall \varepsilon<\varepsilon_{0}$, $\forall n \in \mathbb{N}^{*}$ such that $n<\frac{\mathcal{M}}{\pi}$, there exists a branch of stable solutions of (G.P.) such that:

1) $u\left(\right.$ or $\left.v^{\gamma}\right)$ has exactly $n$ vortices of degree 1 , located at $a_{i}^{\varepsilon}$.

2) $\left|a_{i}^{\varepsilon}\right| \rightarrow 0$ as $\varepsilon \rightarrow 0$, and if we set $\tilde{a_{i}}=a_{i} \sqrt{\omega}$, the $\tilde{a_{i}}$ 's tend to minimize

$$
w\left(x_{1}, \cdots, x_{n}\right)=-\pi \sum_{i \neq j} \log \left|x_{i}-x_{j}\right|+\pi \sum_{i}\left|x_{i}\right|^{2}
$$

so that $\left|a_{i}\right| \leq \frac{C}{\sqrt{\omega}}$, and $\left|a_{i}-a_{j}\right| \geq \frac{C}{\sqrt{\omega}}$.

3) $A s \varepsilon \rightarrow 0$,

$$
J(u)=\frac{\min }{\overline{U_{n}}} J=J(\rho)+\pi n\left(|\log \varepsilon|-R^{2} \omega\right)+\frac{\pi}{2}\left(n^{2}-n\right) \log \omega+w\left(\tilde{a_{1}}, \cdots, \tilde{a_{n}}\right)+Q_{n}+o_{\varepsilon}(1) .
$$

The solution with $n$ vortices minimizes $J$ in $D_{\mathcal{M}}$ exactly for $\omega_{n} \leq \omega \leq \omega_{n+1}$, where $\omega_{n}$ has an expression of the form

$$
\omega_{n}=\frac{|\log \varepsilon|}{R^{2}}+\frac{n-1}{R^{2}}|\log | \log \varepsilon||+O(1) .
$$

Proof. The solutions are given by Lemmas 3.5 and 3.6. Consider $n<\frac{\mathcal{M}}{\pi}$ and $u$ a minimizer of $J$ in $U_{n}$ as given by Lemma 3.6. Then, $u \in U_{n}$ which is open in $H_{0}^{1}$, hence it is a local minimizer of the energy, therefore it is a stable solution of (G.P.). The characteristics of its vortices and its energy are easily derived from the proof of Lemma 3.5. There remains to check that its range of minimality is $\left[\omega_{n}, \omega_{n+1}\right]$. It amounts to computing when $\inf _{\overline{U_{n}}} J<\inf _{\overline{U_{k}}} J, \forall k \neq n$. Comparing the expressions (3.27) for different values of $n$ (exactly as in [17]), we get the desired result.

\subsection{Proof of Lemma 3.1}

The first assertion follows readily from the definition of $T$. For the second one, we begin by expanding $J(u)$ as

$$
\begin{aligned}
J(u) & =\int_{B_{R}} \frac{1}{2}|\nabla u+i u \omega \times x|^{2}+\frac{1}{4 \varepsilon^{2}}\left(1-|u|^{2}\right)^{2} \\
& =\int_{B_{R}} \frac{1}{2}|\nabla u|^{2}+\frac{1}{4 \varepsilon^{2}}\left(1-|u|^{2}\right)^{2}+\frac{\omega^{2}}{2} r^{2}|u|^{2}+\omega\left(i u, x_{2} u_{x_{1}}-x_{1} u_{x_{2}}\right) .
\end{aligned}
$$

Next, we notice that $\rho T(v)=u$ if $|u| \leq 1$ and $\rho T(v)=\frac{u}{|u|}$ if $|u| \geq 1$, hence obvioulsy

$$
\int_{B_{R}}|\nabla(\rho T(v))|^{2} \leq \int_{B_{R}}|\nabla u|^{2}
$$

and

hence

$$
\int_{B_{R}}\left(1-|\rho T(v)|^{2}\right)^{2} \leq \int_{B_{R}}\left(1-|u|^{2}\right)^{2}
$$

$$
F(\rho T(v)) \leq F(u)
$$


Moreover, as in [16], proof of Lemma 2.2,

$$
\begin{gathered}
\Delta:=\left|\int_{B_{R}}\left(i \rho T(v), x_{2}(\rho T(v))_{x_{1}}-x_{1}(\rho T(v))_{x_{2}}\right)-\left(i u, x_{2} u_{x_{1}}-x_{1} u_{x_{2}}\right)\right| \\
=\left.\left|\int_{|u| \geq 1}\left(i \frac{u}{|u|}, x_{2}\left(\frac{u}{|u|}\right)_{x_{1}}-x_{1}\left(\frac{u}{|u|}\right)_{x_{2}}\right)-\right| u\right|^{2}\left(i \frac{u}{|u|}, x_{2}\left(\frac{u}{|u|}\right)_{x_{1}}-x_{1}\left(\frac{u}{|u|}\right)_{x_{2}}\right) \\
-\left(i u, \frac{u}{|u|}\left(x_{2}|u|_{x_{1}}-x_{1}|u|_{x_{2}}\right)\right) \mid
\end{gathered}
$$

where the last term vanishes, hence

$$
\Delta=\left|\int_{|u| \geq 1}\left(1-|u|^{2}\right)\left(i \frac{u}{|u|}, x_{2}\left(\frac{u}{|u|}\right)_{x_{1}}-x_{1}\left(\frac{u}{|u|}\right)_{x_{2}}\right)\right| .
$$

But, we consider $u$ such that $J(u) \leq J(\rho)$, hence, arguing as in the proof of (2.7),

$$
\int_{B_{R}}\left(1-|u|^{2}\right)^{2} \leq C \varepsilon^{4} \omega^{4}+C \varepsilon
$$

and by Cauchy-Schwarz's inequality,

$$
\Delta \leq C\left(\int_{B_{R}}\left(1-|u|^{2}\right)^{2}\right)^{\frac{1}{2}}\left(\int_{|u| \geq 1}\left|\nabla \frac{u}{|u|}\right|^{2}\right)^{\frac{1}{2}} .
$$

On the other hand, $J(u) \leq J(\rho)$ also implies that

$$
\begin{aligned}
\int_{B_{R}} \frac{\rho^{2}}{2}|\nabla v|^{2}+\frac{\rho^{4}}{4 \varepsilon^{2}}\left(1-|v|^{2}\right)^{2} & \leq \omega\left|\int_{B_{R}} \rho^{2}\left(i v, x_{2} v_{x_{1}}-x_{1} v_{x_{2}}\right)\right| \\
\int_{B_{R}} \frac{\rho^{2}}{2}|\nabla v|^{2} & \left.\leq \omega \int_{B_{R}} \rho^{2}|v||\nabla v| \leq \omega\|u\|_{L^{2}\left(B_{R}\right)}\right) \rho \nabla v \|_{L^{2}\left(B_{R}\right)} .
\end{aligned}
$$

But, using (3.37),

Therefore (3.39) implies that

$$
\int_{B_{R}}|u|^{2}=\int_{B_{R}}|u|^{2}-1+\int_{B_{R}} 1 \leq C+C\left(\int_{B_{R}}\left(1-|u|^{2}\right)^{2}\right)^{\frac{1}{2}} \leq C .
$$

$$
\int_{B_{R}} \rho^{2}|\nabla v|^{2} \leq C \omega^{2}
$$

From (3.40), we deduce

$$
\int_{|u| \geq 1}\left|\nabla \frac{u}{|u|}\right|^{2} \leq \int_{\rho|v| \geq 1}\left|\nabla \frac{v}{|v|}\right|^{2} \leq \int_{\rho|v| \geq 1} \rho^{2}|v|^{2}\left|\nabla \frac{v}{|v|}\right|^{2} \leq \int_{B_{R}} \rho^{2}|\nabla v|^{2} \leq C \omega^{2} .
$$

Therefore, combining $(3.41)$ and $(3.37,3.38)$ becomes

$$
\omega \Delta \leq C \omega^{2}\left(\varepsilon^{\frac{1}{2}}+\omega^{2} \varepsilon^{2}\right)=o(1),
$$

as soon as $\omega \ll \varepsilon^{-\frac{1}{4}}$. Combined with (3.35) and (3.36), this allows us to conclude that $J(\rho T(v)) \leq J(\rho v)+o(1)$ for $\omega$ satisfying $\omega \leq C \varepsilon^{-\alpha}$ if we choose $0<\alpha<\frac{1}{4}$. 


\subsection{Proof of Lemma 3.4}

The first step is to prove that

$$
I:=\int_{B_{R}}(i u, d X \wedge d u) \simeq \int_{B^{\prime}}\left(i v^{\gamma}, d X \wedge d v^{\gamma}\right)
$$

Writing $u=\rho v$,

$$
\int_{B_{R}}(i u, d X \wedge d u)=\int_{B_{R}} \rho^{2}(i v, d X \wedge d v)+\int_{B_{R}}(i v, v) d X \wedge d \rho
$$

where the second term vanishes identically. Then, recalling that we assume $\rho|v| \leq 1$,

$$
\begin{aligned}
\left|\int_{B_{R} \backslash B^{\prime}} \rho^{2}(i v, d X \wedge d v)\right| & \leq \int_{B_{R} \backslash B^{\prime}} \rho^{2}|v\|\nabla v\| \nabla X| \leq C\left(\int_{B_{R}}|\nabla v|^{2} \rho^{2}\right)^{\frac{1}{2}}\left(\int_{B_{R} \backslash B^{\prime}} \rho^{2}|v|^{2}\right)^{\frac{1}{2}} \\
& \leq C\|v\|_{H_{\rho^{2}}^{1}}\left(\operatorname{vol}\left(B_{R} \backslash B^{\prime}\right)\right)^{\frac{1}{2}} \leq C|\log \varepsilon|^{\frac{1}{2}} \varepsilon^{\frac{\beta}{2}}
\end{aligned}
$$

where we have used the fact that $u \in D_{\mathcal{M}}$. This term is thus a $o\left(\frac{1}{\omega}\right)$ as soon as $\alpha<\frac{\beta}{2}$. Hence we can replace $I$ by $\int_{B^{\prime}} \rho^{2}(i v, d X \wedge d v)$. Furthermore, as $\rho \geq 1-o(1)$ on $B^{\prime}$,

$$
\left|\int_{B^{\prime}}\left(\rho^{2}-1\right)(i v, d X \wedge d v)\right| \leq C\left(\int_{B_{R}}\left(1-\rho^{2}\right)^{2}\right)^{\frac{1}{2}}\|\nabla v\|_{L^{2}\left(B^{\prime}\right)} \leq C|\log \varepsilon|^{\frac{1}{2}}\left(\varepsilon^{\frac{1}{2}}+\omega^{2} \varepsilon^{2}\right)=o\left(\frac{1}{\omega}\right)
$$

if $\alpha$ is well-chosen. Thus, we can assert that

$$
I=\int_{B^{\prime}}(i v, d X \wedge d v)+o\left(\frac{1}{\omega}\right)
$$

Then, we deduce, following exactly the proof of [16], Lemmas 4.2 and 4.3, that

$$
I=\int_{B^{\prime}}\left(i v^{\gamma}, d X \wedge d v^{\gamma}\right)+o\left(\frac{1}{\omega}\right)=2 \pi \sum_{i} d_{i} X\left(a_{i}\right)+o\left(\frac{1}{\omega}\right)
$$

because we have chosen $X=0$ on $\partial B^{\prime}$. This completes the proof.

\section{Study of Global minimizers of $J$ FOR $\omega \gg|\log \varepsilon|$}

In this section, we return to the general case of $\Omega$ and prove that results similar to those of [21] can be obtained for the functional $J$. We do not use the approach of local minimization any longer, but study minimizers of $J$ over all $H_{0}^{1}$ and evaluate their energy as a function of $\omega$. We start with the particular case $\lambda=0$, i.e. $\omega \gg|\log \varepsilon|$. The case $\lambda>0$ is treated in Section 5, and can be read independently.

\subsection{An upper bound for the minimal energy}

Following exactly the same method as in [21], we prove the following proposition:

Proposition 4.1. Assume $\omega \rightarrow+\infty$ and $\omega \ll \frac{1}{\varepsilon}$. Then, as $\varepsilon \rightarrow 0$,

$$
\min _{H_{0}^{1}(\Omega, \mathbb{C})} J \leq F(\rho)+\omega|\Omega| \log \frac{1}{\varepsilon \sqrt{\omega}}+O(\omega),
$$

where $|$.$| denotes the volume. If we only assume \omega \ll \frac{1}{\varepsilon^{2}}$, then as $\varepsilon \rightarrow 0$, $\min _{H_{0}^{1}(\omega, \mathbb{C})} J \leq F(\rho)+o\left(\omega^{2}\right)$. 
The proof consists, as in [21], in constructing a test configuration and evaluating its energy. We only state the main steps.

The idea of the proof comes from the following facts. We notice that, if $u$ is solution of (G.P.), then the following equation is satisfied, letting $u=\eta e^{i \varphi}$,

$$
(-\Delta u, i u)=\left(\frac{u}{\varepsilon^{2}}\left(1-|u|^{2}\right)+2 i \nabla u . \omega \times x-\omega^{2} r^{2} u, i u\right),
$$

thus, if we define this time $X$ by

we have

$$
X=\frac{|x|^{2}}{2}
$$

$$
\begin{gathered}
\operatorname{div}(\nabla u, i u)=\omega \nabla \eta^{2} \cdot \nabla^{\perp} X, \\
\operatorname{div}\left(\eta^{2}\left(\nabla \varphi-\omega \nabla^{\perp} X\right)\right)=0,
\end{gathered}
$$

where $\eta^{2} \nabla \varphi$ is to be understood as $(\nabla u, i u)$ hence has a meaning everywhere. Thus, as $\Omega$ is simply connected, we can write

$$
\eta^{2}\left(\nabla \varphi-\omega \nabla^{\perp} X\right)=\nabla^{\perp} U
$$

for some function $U \in H^{1}(\Omega, \mathbb{C})$. Actually, the proof can be adjusted to non-simply connected domains as in [21]. Moreover, as $\eta=0$ on $\partial \Omega, \nabla U=0$ on $\partial \Omega$, hence $U$ is constant on $\partial U$, and since it is defined up to a constant, we can choose to take $U=0$ on $\partial \Omega$ :

$$
\begin{cases}\eta^{2}\left(\nabla \varphi-\omega \nabla^{\perp} X\right)=\nabla^{\perp} U & \text { in } \Omega \\ U=0 & \text { on } \partial \Omega .\end{cases}
$$

Here, $U$ plays a role similar to that of the induced magnetic field $h$ in [21]. Then, one notices, as in [21], that

$$
\int_{\Omega}|\nabla u+i \omega \times x u|^{2}=\int_{\Omega}|\nabla \eta|^{2}+\eta^{2}\left|\nabla \varphi-\omega \nabla^{\perp} X\right|^{2}=\int_{\Omega}|\nabla \eta|^{2}+\frac{|\nabla U|^{2}}{\eta^{2}} \geq \int_{\Omega}|\nabla \eta|^{2}+|\nabla U|^{2} .
$$

The idea of the proof is to construct $U$ as in [21], and to deduce the phase $\varphi$ by (4.4), so that $u$ has vortices of degree 1 , regularly set on a lattice in $\Omega$.

\section{Proof of the proposition.}

Step 1: Let $K$ be a square centered at 0 , of edge-size $\sqrt{\frac{\pi}{\omega}}$. Defining

$$
\begin{cases}\mu=\frac{2}{\varepsilon^{2}} & \text { in } B(0, \varepsilon) \subset K \\ \mu=0 & \text { otherwise in } K\end{cases}
$$

we choose $U$ to be the solution of

$$
\begin{cases}\Delta U=\mu-2 \omega & \text { in } K \\ \frac{\partial U}{\partial n}=0 & \text { on } \partial K\end{cases}
$$

This problem has a solution because

$$
\int_{K}(\mu-2 \omega)=2 \pi-2 \omega|K|=0
$$


The solution can indeed be found by minimizing

$$
\int_{K}|\nabla U|^{2}+\int_{K}(\mu-2 \omega) U
$$

over all $U$ such that $\int_{K} U=0$. As in [21], in order to compute the energy of $U$, we use the following comparison $\operatorname{map}$ in $D=B\left(0, \sqrt{\frac{\pi}{\omega}}\right)$ :

$$
\begin{cases}\Delta f=\mu-2 \omega & \text { in } D \\ f=0 & \text { on } \partial D\end{cases}
$$

As in [21], we prove that $f$ is radial, and $\forall r \in\left[\varepsilon, \sqrt{\frac{\pi}{\omega}}\right]$,

$$
f^{\prime}(r) 2 \pi r=\int_{\partial B(0, r)} \frac{\partial f}{\partial n}=\int_{B(0, r)} \Delta f=\int_{B(0, r)} \mu-2 \omega=2 \pi-2 \omega \pi r^{2}
$$

so that

$$
f^{\prime}(r)=\frac{1}{r}-\omega r
$$

and similarly, for $r \leq \varepsilon$, in view of (4.6),

$$
f^{\prime}(r)=\frac{r}{\varepsilon^{2}}-\omega r
$$

From (4.10) and (4.11), we check that

$$
\int_{D}|\nabla f|^{2} \leq C+2 \pi \log \frac{1}{\varepsilon \sqrt{\omega}}
$$

Step 2: Comparison of $U$ and $f$.

Defining $g=U-f$, we have

$$
\begin{cases}\Delta g=0 & \text { in } K \\ \frac{\partial g}{\partial n}=-\frac{\partial f}{\partial n} & \text { on } \partial K\end{cases}
$$

Exactly the same proof as in [21], Lemma 3.2 can be reproduced (it uses essentially the scaled trace theorem and Poincaré-Wirtinger inequality), to obtain that $\|\nabla g\|_{L^{2}(K)} \leq C$. Then, obviously,

$$
\int_{K}|\nabla U|^{2} \leq C+2 \pi \log \frac{1}{\varepsilon \sqrt{\omega}} .
$$

Step 3: Extension of $U$ and definition of $u$.

As in [21], we cover $\Omega$ with a square lattice of period $\sqrt{\frac{\pi}{\omega}}$, and we extend $U$ and $\mu$ by periodicity with respect to this lattice. We denote $a_{i}$ the centers of the squares. There are $\simeq \frac{|\Omega| \omega}{\pi}$ of them. This leads to a $U \in H^{1}$ because $\frac{\partial U}{\partial n}=0$ on $\partial K$, and

$$
\frac{1}{2} \int_{\Omega}|\nabla U|^{2} \leq|\Omega| \omega \log \frac{1}{\varepsilon \sqrt{\omega}}+O(\omega)
$$


Then, we define

$$
\nabla \varphi=\nabla^{\perp} U+\omega \nabla^{\perp} X \quad \text { in } \Omega \backslash \cup_{i} B\left(a_{i}, \varepsilon\right)
$$

and choose $\eta$ that satisfies

$$
\left\{\begin{array}{l}
\eta \equiv 0 \text { in } B\left(a_{i}, \varepsilon\right) \\
\eta \equiv 1 \text { in } \Omega \backslash \cup_{i} B\left(a_{i}, 2 \varepsilon\right) \\
\eta \leq 1 \\
\frac{1}{2} \int_{B\left(a_{i}, 2 \varepsilon\right)}|\nabla \eta|^{2}+\frac{1}{2 \varepsilon^{2}}\left(1-\eta^{2}\right)^{2} \leq C \\
\int_{B\left(a_{i}, 2 \varepsilon\right)}\left(1-\eta^{2}\right) \leq C \varepsilon^{2} .
\end{array}\right.
$$

$u$ is finally defined by

$$
u=\rho \eta e^{i \varphi} .
$$

This has a meaning because, considering $V$, any subset of $\Omega \backslash \cup_{i} B\left(a_{i}, \varepsilon\right)$,

$$
\int_{\partial V} \frac{\partial \varphi}{\partial \tau}=\int_{\partial V} \frac{\partial U}{\partial n}+\omega \frac{\partial X}{\partial n}=\int_{V} \Delta U+\omega \Delta X=\int_{V} \Delta U+2 \omega=\int_{V} \mu \in 2 \pi \mathbb{Z}
$$

in view of the definition of $\mu$ (4.6), and (4.7). In addition, in $\cup_{i} B\left(a_{i}, \varepsilon\right), \eta \equiv 0$ so $u$ is well-defined there too.

Step 4: Energy of $u$.

We have all the elements to bound $J(u)$ from above. We denote as usual $v=\frac{u}{\rho}=\eta e^{i \varphi}$. Starting from (2.12), and translating the lattice as in [21] if necessary, we have, using $\rho \leq 1$ (4.16) and (4.17),

$$
\begin{aligned}
J(u) & =J(\rho)+\int_{\Omega} \frac{\rho^{2}}{2}|\nabla v|^{2}+\frac{\rho^{4}}{4 \varepsilon^{2}}\left(1-|v|^{2}\right)^{2}+\int_{\Omega} \omega \rho^{2}(i v, d v \wedge d X) \\
& =J(\rho)-\frac{\omega^{2}}{2} \int_{\Omega} \rho^{2} r^{2}|v|^{2}+\frac{1}{2} \int_{\Omega} \rho^{2}\left|\nabla v-i \omega v \nabla^{\perp} X\right|^{2}+\int_{\Omega} \frac{\rho^{4}}{4 \varepsilon^{2}}\left(1-|v|^{2}\right)^{2} \\
& =J(\rho)-\frac{\omega^{2}}{2} \int_{\Omega} \rho^{2} r^{2}+\frac{1}{2} \int_{\Omega} \rho^{2}\left(|\nabla \eta|^{2}+\eta^{2}|\nabla U|^{2}\right)+\frac{\rho^{4}}{4 \varepsilon^{2}}\left(1-\eta^{2}\right)^{2}+\frac{\omega^{2}}{2} \rho^{2} r^{2}\left(1-\eta^{2}\right) .
\end{aligned}
$$

Since there are $O(\omega)$ squares, from (4.17), we have

$$
\frac{1}{2} \int_{\Omega}|\nabla \eta|^{2}+\frac{1}{2 \varepsilon^{2}}\left(1-\eta^{2}\right)^{2} \leq C \omega
$$

and $\int_{\Omega}(1-\eta) \leq C \varepsilon^{2} \omega$. Thus

since $\varepsilon \omega \rightarrow 0$. Thus, using (4.15),

$$
\left|\frac{\omega^{2}}{2} \int_{\Omega} \rho^{2} r^{2}\left(1-\eta^{2}\right)\right| \leq C \varepsilon^{2} \omega^{3}=o(\omega)
$$

$$
\begin{aligned}
J(u) & \leq J(\rho)-\frac{\omega^{2}}{2} \int_{\Omega} \rho^{2} r^{2}+\frac{1}{2} \int_{\Omega}|\nabla U|^{2}+\int_{\Omega} \frac{1}{2}|\nabla \eta|^{2}+\frac{1}{4 \varepsilon^{2}}\left(1-\eta^{2}\right)^{2}+O\left(\omega^{3} \varepsilon^{2}\right) \\
& \leq J(\rho)-\frac{\omega^{2}}{2} \int_{\Omega} \rho^{2} r^{2}+|\Omega| \omega \log \frac{1}{\varepsilon \sqrt{\omega}}+O(\omega) .
\end{aligned}
$$


Furthermore, as

we can also write

$$
J(\rho)=\frac{1}{2} \int_{\Omega}|\nabla \rho|^{2}+\frac{1}{2 \varepsilon^{2}}\left(1-\rho^{2}\right)^{2}+\omega^{2} r^{2} \rho^{2}=F(\rho)+\frac{1}{2} \omega^{2} \int_{\Omega} r^{2} \rho^{2},
$$

$$
J(u) \leq F(\rho)+|\Omega| \omega \log \frac{1}{\varepsilon \sqrt{\omega}}+O(\omega)
$$

If one removes the assumption $\omega \ll \frac{1}{\varepsilon}$, and replaces it by $\omega \ll \frac{1}{\varepsilon^{2}}$, we get at least $J(u) \leq F(\rho)+o\left(\omega^{2}\right)$. This proves the proposition.

Proposition 4.2. If $|\log \varepsilon| \ll \omega \ll \frac{1}{\varepsilon}$, then

$$
J(\rho)-\frac{\omega^{2}}{2} \int_{\Omega} r^{2}+\omega|\Omega| \log \frac{1}{\varepsilon \sqrt{\omega}}(1+o(1)) \leq \min _{H_{0}^{1}(\Omega, \mathbb{C})} J \leq F(\rho)+\omega|\Omega| \log \frac{1}{\varepsilon \sqrt{\omega}}(1+o(1)) .
$$

Proof. This proof is essentially the same as that of Section 4 in [21]. We explain how to obtain it by modifying step by step the proof of [21]. $h_{\mathrm{ex}}$ will always be replaced by $\omega$. We consider $u$ a minimizer of $J$, we then know that $|u| \leq 1$ by the maximum principle.

Step 1: We split the energy as in Lemma 2.2, using $v=\frac{u}{\rho}$ :

$$
J(u)=J(\rho)+\int_{\Omega} \frac{\rho^{2}}{2}|\nabla v|^{2}+\frac{\rho^{4}}{4 \varepsilon^{2}}\left(1-|v|^{2}\right)^{2}-\omega \int_{\Omega} \rho^{2}\left(i v, \nabla v \cdot \nabla^{\perp} X\right) .
$$

Writing as usual $v=|v| e^{i \varphi}(4.18)$ can be transformed into

$$
J(u)=J(\rho)+\frac{1}{2} \int_{\Omega} \rho^{2}\left|\nabla v-i \omega v \nabla^{\perp} X\right|^{2}+\frac{\rho^{4}}{4 \varepsilon^{2}}\left(1-|v|^{2}\right)^{2}-\frac{\omega^{2}}{2} \int_{\Omega} \rho^{2}|v|^{2} r^{2} .
$$

Using that $\rho|v|=|u| \leq 1$, we may write

$$
J(u) \geq J(\rho)-\int_{\Omega} \frac{\omega^{2}}{2} r^{2}+\frac{1}{2} \int_{\Omega} \rho^{2}\left|\nabla v-i \omega v \nabla^{\perp} X\right|^{2}+\frac{\rho^{4}}{4 \varepsilon^{2}}\left(1-|v|^{2}\right)^{2} .
$$

We are going to study the last integral as in [21]. Setting as previously $\Omega^{\prime}=\Omega \backslash\left\{x / \delta(x) \leq \varepsilon^{\beta}\right\}$, where $\left.\beta \in\right] 0,1$, we have, from $(2.5)$,

$$
0 \leq 1-\rho \leq o(1) \quad \text { in } \Omega^{\prime}
$$

We now restrict to studying

$$
j(u):=\int_{\Omega^{\prime}} \frac{1}{2}\left|\nabla v-i \omega v \nabla^{\perp} X\right|^{2}+\frac{1}{4 \varepsilon^{2}}\left(1-|v|^{2}\right)^{2},
$$

because we can assert that

$$
J(u) \geq J(\rho)-\int_{\Omega} \frac{\omega^{2}}{2} r^{2}+(1-o(1)) j(u) .
$$

Step 2: As in [21], we partition $\Omega^{\prime}$ into disjoint squares $K$ whose centers are on a square lattice, of size $\delta(\varepsilon)=$ $o(1)$, to be chosen as in [21] in order to satisfy

$$
L \ll \omega \delta^{2} \ll \min \left(\omega, L^{2}\right) \quad \text { with } L:=\log \frac{1}{\varepsilon \sqrt{\omega}}
$$


We distinguish between the "good squares" on which

$$
j_{K}(u) \leq 2 \delta^{2} \omega L
$$

and the "bad squares" on which

$$
j_{K}(u)>2 \delta^{2} \omega L
$$

$j_{K}$ here denotes the restriction of $j$ to $K$.

We need to prove a lower bound on the good squares only, since we already have one on the bad squares. From now on, $K$ denotes a "good square".

Step 3: We denote $\Omega_{t}=\{x \in K /|v(x)|<t\}, \gamma_{t}=\partial \Omega_{t}$. Using $\int_{K}\left|\nabla v-i v \omega \nabla^{\perp} X\right|^{2}=\int_{K}|\nabla| v||^{2}+|v|^{2} \mid \nabla \varphi$ $-\left.\omega \nabla^{\perp} X\right|^{2}$ and applying the co-area formula as in [21], Section 4.2, we obtain the following result,

$$
\begin{aligned}
j_{K}(u) \geq \int_{0}^{\infty} a(t)+2 t b(t) d t \geq \int_{0}^{1} a(t)+2 t b(t) d t \\
a(t):=\int_{\gamma_{t} \cap K} \frac{|\nabla| v||}{2}+\frac{\left(1-t^{2}\right)^{2}}{4 \varepsilon^{2}|\nabla| v||} \\
b(t):=\frac{1}{2} \int_{K \backslash \overline{\Omega_{t}}}\left|\nabla \varphi-\omega \nabla^{\perp} X\right|^{2} .
\end{aligned}
$$

This will provide the desired lower bound.

Lemma 4.2 of [21] can be reproduced without change. We will show in Steps 5 and 6 that the result of Lemma 4.3 of [21] remains valid.

Step 4: End of the proof of the proposition.

We deduce as in Section 4.3 of [21] the similar lower bound on our good square:

$$
j_{K}(u) \geq \omega \delta^{2} L(1-o(1)) .
$$

This lower bound is also true on the bad squares from (4.26). If $N(\varepsilon)$ is the total number of squares included in $\Omega^{\prime}, N(\varepsilon) \delta^{2} \rightarrow\left|\Omega^{\prime}\right|$ as $\varepsilon \rightarrow 0$, since $\delta \rightarrow 0$. Hence, adding up all the lower bounds, we are led to

$$
j(u) \geq\left|\Omega^{\prime}\right| \omega L(1-o(1)),
$$

and as $\left|\Omega^{\prime}\right|=|\Omega|-o(1)$, we deduce with (4.23),

$$
J(u) \geq J(\rho)-\int_{\Omega} \frac{\omega^{2}}{2} r^{2}+(1-o(1))|\Omega| \omega \log \frac{1}{\varepsilon \sqrt{\omega}},
$$

which proves Proposition 4.2 and Theorem 3. The conclusion in the case $\omega \leq \frac{1}{\varepsilon^{\frac{4}{5}}}$ follows from (2.7).

Step 5: We show how to adjust the proof of Lemma 4.3 of [21]. First, we need to adjust the proof of Proposition 4.1 in [21], which relies on Lemma 4.4. For this lemma, juste replace the calculation by the following:

$$
\forall t \in[r, R] \quad 2 \pi d=\int_{\partial B_{t}} \frac{\partial \varphi}{\partial \tau}=\int_{\partial B_{t}} \frac{\partial \varphi}{\partial \tau}-\omega \frac{\partial X}{\partial n}+\int_{\partial B_{t}} \omega \frac{\partial X}{\partial n}=\int_{\partial B_{t}} \frac{\partial \varphi}{\partial \tau}-\omega \frac{\partial X}{\partial n}+\int_{B_{t}} 2 \omega
$$


since $\Delta X=2$. Now, using the Cauchy-Schwarz inequality

$$
\int_{\partial B_{t}}\left|\frac{\partial \varphi}{\partial \tau}-\omega \frac{\partial X}{\partial n}\right|^{2} \geq \frac{1}{2 \pi t}\left(\int_{\partial B_{t}} \frac{\partial \varphi}{\partial \tau}-\omega \frac{\partial X}{\partial n}\right)^{2} \geq \frac{1}{2 \pi t}\left(2 \pi d-2 \pi \omega t^{2}\right)^{2}
$$

with (4.31). Using the notation $e(t)=\frac{1}{2} \int_{\partial B_{t}}\left|\frac{\partial \varphi}{\partial \tau}-\omega \frac{\partial X}{\partial n}\right|^{2}$ as in [21], we get that

$$
e(t) \geq \frac{\pi|d|}{t}-2 \pi|d| \omega t
$$

which leads to the same result as in [21] if we replace $f(r, R)$ by $\pi\left(\log \frac{R}{r}-\omega\left(R^{2}-r^{2}\right)\right)$. This new $f$ also satisfies the results of Lemma 4.5 of [21]. The rest of the proof of Proposition 4.1 is identical and yields to balls $B_{i}$, with lower bounds

$$
\frac{1}{2} \int_{B_{i} \backslash V}\left|\nabla \varphi-\omega \nabla^{\perp} X\right|^{2} \geq \pi\left|d_{i}\right|\left(\log \frac{\sigma}{r(V)}-C\right)_{+}
$$

with the same notations as in Proposition 4.1 of [21], except that $\omega$ is replaced by $V$.

Step 6: We adjust the rest of the proof of Lemma 4.3. The lower bound (4.22) on $b(t)$ is still true (with $\omega$ instead of $\left.h_{\mathrm{ex}}\right)$. "Step 1: an auxiliary field" has to be suppressed and "Step 2: estimating $d_{t}$ " to be modified as follows. As in (4.4), we can define a function $U$ on our square $K$ such that

$$
\eta^{2}\left(\nabla \varphi-\omega \nabla^{\perp} X\right)=\nabla^{\perp} U
$$

As in (4.5), since we are on a good square

$$
\begin{aligned}
4 \omega^{2} \delta^{2} L \geq 2 j_{K}(u) & \geq \int_{\Omega}|\nabla| v||^{2}+|v|^{2}\left|\nabla \varphi-\omega \nabla^{\perp} X\right|^{2} \geq\left.\int_{\Omega}|\nabla| v\right|^{2}+\frac{|v|^{2}}{\eta^{4}}|\nabla U|^{2} \\
& =\left.\int_{\Omega}|\nabla| v\right|^{2}+\frac{1}{\eta^{2} \rho^{2}}|\nabla U|^{2} \geq \int|\nabla \eta|^{2}+|\nabla U|^{2},
\end{aligned}
$$

hence we have a good control on $U$. Therefore, replacing $\bar{h}$ by $U$, we can find as in [21] some $t_{0}$ satisfying the equivalent of (4.31):

$$
\partial K_{t_{0}} \cap \cup_{i} B_{i}=\varnothing \quad \text { and } \quad \int_{\partial K_{t_{0}}}\left|\frac{\partial U}{\partial n}\right|^{2}<\frac{8 \omega \delta^{2}}{\alpha t_{0}^{2}} L
$$

Consequently, as in (4.32) in [21],

$$
2 \pi d_{t} \geq 2 \pi \sum_{i} d_{B_{i}}=\int_{\partial K_{t_{0}}} \frac{\partial \varphi}{\partial \tau}=\int_{\partial K_{t_{0}}} \frac{1}{\eta^{2}} \frac{\partial U}{\partial n}+\omega \frac{\partial X}{\partial n}=\int_{K_{t_{0}}} 2 \omega+\int_{\partial K_{t_{0}}} \frac{1}{\eta^{2}} \frac{\partial U}{\partial n} .
$$

The second term can be bounded from above, using the fact that $\partial K_{t_{0}} \cap \cup_{i} B_{i}=\varnothing$, hence $\eta>t$ on $\partial K_{t_{0}}$ :

$$
\left|\int_{\partial K_{t_{0}}} \frac{1}{\eta^{2}} \frac{\partial U}{\partial n}\right| \leq \frac{1}{t^{2}}\left(\int_{\partial K_{t_{0}}}\left|\frac{\partial U}{\partial n}\right|^{2}\right)^{\frac{1}{2}} \sqrt{\delta} \leq \frac{C \delta^{\frac{3}{2}}}{t^{2}}\left(\frac{\omega L}{\alpha}\right)^{\frac{1}{2}}
$$


using (4.34). The first term is exactly $2 \omega t_{0}^{2}$, and using the estimates on $t_{0}$, we obtain the exact analogue of (4.33) of $[21]$ :

$$
2 \pi d_{t} \geq 2 \omega \delta^{2}\left(1-\frac{4 \alpha}{\delta}-\frac{C}{t}\left(\frac{L}{\omega \delta \alpha}\right)^{\frac{1}{2}}\right)
$$

We are led to

$$
2 \pi d_{t} \geq 2 \omega \delta^{2}\left(1-\frac{C}{t} \Delta\right)_{+},
$$

then, the rest of the proof can be exactly reproduced. Notice that the only change is the factor 2 coming from $\Delta X$ that appears in (4.36) above and leads to twice the lower bound of [21]. This completes the proof of the proposition and of Theorem 3.

We recall Theorem 3:

Theorem 3. Assume $|\log \varepsilon| \ll \omega \ll \frac{1}{\varepsilon}$. Then

$$
J(\rho)-\int_{\Omega} \frac{\omega^{2}}{2} r^{2}+\omega|\Omega| \log \frac{1}{\varepsilon \sqrt{\omega}}(1-o(1)) \leq \min _{H_{0}^{1}(\Omega, \mathbb{C})} J \leq F(\rho)+\omega|\Omega| \log \frac{1}{\varepsilon \sqrt{\omega}}+O(\omega),
$$

where $|$.$| denotes the volume.$

If in addition $\omega \leq \frac{C}{\varepsilon^{4 / 5}}$, then

$$
\min _{H_{0}^{1}(\Omega, \mathbb{C})} J=F(\rho)+\omega|\Omega| \log \frac{1}{\varepsilon \sqrt{\omega}}(1+o(1)) .
$$

Reproducing the proof of Section 5 of [21], we can obtain the same result concerning the existence of large vortex-balls in $\Omega^{\prime}$ :

Theorem 4. Let $|\log \varepsilon| \ll \omega \leq \frac{C}{\varepsilon^{4 / 5}}$, and $u_{\varepsilon}$ be a corresponding minimizer of $J$. Then, for $\varepsilon<\varepsilon_{0}$, there exists a family of disjoint disks $\left(B_{i}^{\varepsilon}\right)$ with radii each less than $\frac{1}{\sqrt{\omega}}$ and sum less than $|\Omega| \sqrt{\omega}$, such that $\left|u_{\varepsilon}\right| \geq \frac{1}{2}$ on $\partial B_{i}^{\varepsilon}$ and, if $a_{i}^{\varepsilon}$ is the center of $B_{i}^{\varepsilon}$ and $d_{i}^{\varepsilon}=\operatorname{deg}\left(\frac{u_{\varepsilon}}{\left|u_{\varepsilon}\right|}, \partial B_{i}^{\varepsilon}\right)$, then

$$
\mu_{\varepsilon}=\frac{2 \pi}{\omega} \sum_{i} d_{i}^{\varepsilon} \delta_{a_{i}^{\varepsilon}} \underset{\varepsilon \rightarrow 0}{\longrightarrow} 2 d x
$$

in the weak sense of measures, where $d x$ is the Lebesgue measure on $\mathbb{R}^{2}$ restricted to $\Omega$.

Moreover,

$$
\pi \sum_{i}\left|d_{i}^{\varepsilon}\right| \simeq \pi \sum_{i} d_{i}^{\varepsilon} \simeq \omega|\Omega|
$$

and most of the vortex-energy is concentrated in the balls, i.e.

$$
J_{\Omega \backslash \cup_{i} B_{i}^{\varepsilon}}\left(u_{\varepsilon}\right)-F(\rho)=o\left(J\left(u_{\varepsilon}\right)-F(\rho)\right) .
$$

\section{Global minimizers in the general Case: The free-Boundary model}

In this section, we will consider $\omega$ 's such that $\omega \leq O(|\log \varepsilon|)$ i.e. the intermediate case for which the results of Section 4 are not relevant. More precisely, similarly as in [22], we assume that

$$
\lambda=\lim _{\varepsilon \rightarrow 0} \frac{|\log \varepsilon|}{\omega(\varepsilon)}
$$


exists and is finite, positive. We recall that $\mathcal{R}$ denotes the set of Radon measures on $\Omega$, and we define

$$
E(f)=\frac{\lambda}{2} \int_{\Omega}|\Delta f+2|+\frac{1}{2} \int_{\Omega}|\nabla f|^{2}
$$

over the set

$$
V=\left\{f \in H_{0}^{1}(\Omega) / \Delta f \in \mathcal{R}\right\} .
$$

\subsection{Upper bound for the minimal energy}

We have the following:

Proposition 5.1. Let $\omega(\varepsilon)$ be such that $\lambda>0$ and $u_{\varepsilon}$ be a minimizer of $J$, then

$$
\limsup _{\varepsilon \rightarrow 0} \frac{J\left(u_{\varepsilon}\right)-F(\rho)}{\omega^{2}} \leq \min _{V} E
$$

We shall see later that $\min _{V} E$ is uniquely achieved by some function $U_{*}$ for which $\mu_{*}=\Delta U_{*}+2$ is a positive measure, and an $L^{\infty}(\Omega)$ function.

We do not write the proof of this proposition in full, because it is mainly the same as in [22]. One only has to replace the definition of $G$ by the Green kernel

$$
\begin{cases}-\Delta_{x} G(x, y)=\delta_{y} & \text { in } \Omega \\ G(x, y)=0 & \text { on } \partial \Omega .\end{cases}
$$

We construct as in Proposition 2.2 of [22] a family of $O(\omega)$ points $a_{i}^{\varepsilon}$, and a family of measures $\mu_{\varepsilon}$ supported on the disjoint circles $C\left(a_{i}^{\varepsilon}, \varepsilon\right)$, such that $\mu_{\varepsilon}$ tends to $\mu_{*}$ in $\mathcal{R}$, where $\mu_{*}$ is the measure associated to the minimizer $U_{*}$ of $E$. We define a test-function $U_{\varepsilon}$ by

$$
\begin{cases}\Delta U_{\varepsilon}=\omega\left(\mu_{\varepsilon}-2\right) & \text { in } \Omega \\ U_{\varepsilon}=0 & \text { on } \partial \Omega\end{cases}
$$

Then, it is easy to adjust the proof of [22] in order to get

$$
\begin{aligned}
\frac{1}{2 \omega^{2}} \int_{\Omega}\left|\nabla U_{\varepsilon}\right|^{2} & =\frac{1}{2} \int_{\Omega \times \Omega} G(x, y) d\left(\mu_{\varepsilon}-2\right)(x) d\left(\mu_{\varepsilon}-2\right)(y) \\
& \leq \frac{\lambda}{2} \int_{\Omega} \mu_{*}+\frac{1}{2} \int_{\Omega \times \Omega} G(x, y) d\left(\mu_{*}-2\right)(x) d\left(\mu_{*}-2\right)(y)+o(1) \\
& \leq \frac{\lambda}{2} \int_{\Omega} \mu_{*}+\frac{1}{2} \int_{\Omega}\left|\nabla U_{*}\right|^{2}+o(1) .
\end{aligned}
$$

Thus,

$$
\frac{1}{2 \omega^{2}} \int_{\Omega}\left|\nabla U_{\varepsilon}\right|^{2} \leq E\left(U_{*}\right)+o(1)
$$

Then, as for Proposition 4.1, we construct our test-function by choosing

$$
\nabla \varphi=\nabla^{\perp} U_{\varepsilon}+\omega \nabla^{\perp} X \quad \text { in } \Omega \backslash \cup_{i} B\left(a_{i}^{\varepsilon}, \varepsilon\right) .
$$


The measures $\mu_{\varepsilon}$ were defined so that the mass of $\omega \mu_{\varepsilon}$ restricted to each circle $C\left(a_{i}, \varepsilon\right)$ is exacly $2 \pi$; therefore, if $V$ is any regular subset of $\Omega \backslash \cup_{i} B\left(a_{i}, \varepsilon\right)$, we have

$$
\int_{\partial V} \frac{\partial \varphi}{\partial \tau}=\int_{\partial V} \frac{\partial U_{\varepsilon}}{\partial n}+\omega \frac{\partial X}{\partial n}=\int_{V} \Delta U_{\varepsilon}+\omega \Delta X=\int_{V} \Delta U+2 \omega=\int_{V} \omega \mu_{\varepsilon} \in 2 \pi \mathbb{Z} .
$$

Hence, $e^{i \varphi}$ can be well-defined on $\Omega \backslash \cup_{i} B\left(a_{i}, \varepsilon\right)$. Then, we choose $\eta$ satisfying the same conditions as in (4.17), and define the test-function $u$ to be $u=\rho \eta e^{i \varphi}$. Again, as in (4.19), denoting $v=\frac{u}{\rho}=\eta e^{i \varphi}$,

$$
J(u)=J(\rho)+\frac{1}{2} \int_{\Omega} \rho^{2}\left|\nabla v-i \omega v \nabla^{\perp} X\right|^{2}+\frac{\rho^{4}}{4 \varepsilon^{2}}\left(1-|v|^{2}\right)^{2}-\frac{\omega^{2}}{2} \int_{\Omega} \rho^{2}|v|^{2} r^{2} .
$$

Hence, using $\rho \leq 1, \rho \eta \leq 1$, and (5.7),

$$
\begin{aligned}
J(u) & \leq J(\rho)-\frac{\omega^{2}}{2} \int_{\Omega} \rho^{2} r^{2}+\frac{1}{2} \int_{\Omega}|\nabla \eta|^{2}+|\nabla U|^{2}+\frac{1}{2 \varepsilon^{2}}\left(1-\eta^{2}\right)^{2}+\frac{\omega^{2}}{2} \int_{\Omega} \rho^{2} r^{2}\left(1-|v|^{2}\right) \\
& \leq F(\rho)+\frac{1}{2} \int_{\Omega}|\nabla U|^{2}+\frac{1}{2} \int_{\Omega}|\nabla \eta|^{2}+\frac{1}{2 \varepsilon^{2}}\left(1-\eta^{2}\right)^{2}+\frac{\omega^{2}}{2} \int_{\Omega} \rho^{2} r^{2}\left(1-|v|^{2}\right) .
\end{aligned}
$$

From the conditions (4.17), since there are $O(\omega)$ balls, $\frac{1}{2} \int_{\Omega}|\nabla \eta|^{2}+\frac{1}{2 \varepsilon^{2}}\left(1-\eta^{2}\right)^{2} \leq O(\omega)$, and $\omega^{2} \int_{\Omega}\left(1-|v|^{2}\right)$ $\leq \varepsilon^{2} \omega^{3}$. Hence, using (5.6),

and the proposition is proved.

$$
J(u) \leq F(\rho)+\omega^{2} E\left(U_{*}\right)+O(\omega)
$$

\subsection{Constructing vortex-balls}

From now on, we consider $u_{\varepsilon}$ minimizing $J$, and we associate to it $U_{\varepsilon}$ defined by (1.10). Here we prove the following proposition stated in the introduction:

Proposition 5.2. If $\lambda>0$, there exists $\varepsilon_{0}$ such that for $\varepsilon<\varepsilon_{0}$, if $u_{\varepsilon}$ minimizes $J$, and $U_{\varepsilon}$ is associated to $u_{\varepsilon}$ by (1.10), then there exists a family of balls (depending on $\varepsilon)\left(B_{i}\right)_{i \in I_{\varepsilon}}=\left(B\left(a_{i}, r_{i}\right)\right)_{i \in I_{\varepsilon}}$ satisfying

$$
\begin{aligned}
& \left\{x \in \Omega^{\prime} /|| v|-1| \geq \frac{1}{|\log \varepsilon|}\right\} \subset \cup_{i \in I_{\varepsilon}} B_{i} \\
& \sum_{i \in I_{\varepsilon}} r_{i} \leq \frac{1}{|\log \varepsilon|^{6}} \\
& \frac{1}{2} \int_{B_{i}}\left|\nabla U_{\varepsilon}\right|^{2} \geq \pi\left|d_{i}\right||\log \varepsilon|(1-o(1))
\end{aligned}
$$

where $d_{i}=\operatorname{deg}\left(u, \partial B_{i}\right)$.

As in Section 3, $\Omega^{\prime}$ denotes $\left\{x \in \Omega / \delta(x) \geq \varepsilon^{\beta}\right\}$.

Proof. As in Proposition 4.2, we define $v=\frac{u}{\rho}, \Omega_{t}=\left\{x \in \Omega^{\prime} /|v(x)|<t\right\}$, and $\gamma_{t}=\partial \Omega_{t}$. From the $a$ priori upper bound of Proposition 5.1, $J(u)-F(\rho) \leq C \omega^{2}$, hence

$$
\frac{1}{2} \int_{\Omega} \rho^{2}|\nabla v|^{2}+\frac{\rho^{4}}{2 \varepsilon^{2}}\left(1-|v|^{2}\right)^{2} \leq C \omega^{2}
$$


and

$$
\frac{1}{2} \int_{\Omega^{\prime}}|\nabla v|^{2}+\frac{1}{2 \varepsilon^{2}}\left(1-|v|^{2}\right)^{2} \leq C \omega^{2} .
$$

As in [22], Proposition 1.1, applying the coarea formula to (5.11), we can find $t \in\left[1-\frac{1}{|\log \varepsilon|}, 1+\frac{1}{\mid \log \varepsilon}\right]$, such that $r\left(\gamma_{t}\right) \leq C \varepsilon|\log \varepsilon|^{3}$. (As in [21], $r\left(\gamma_{t}\right)$ is defined as the infimum over all finite coverings of $\gamma_{t}$ by balls, of the sum of the radii of the balls.) Arguing as for (4.32) with $\sigma=\frac{1}{|\log \varepsilon|^{6}}$ and $V=\Omega_{t}$, we can find balls $B_{i}$ covering $\Omega_{t}$ such that

$$
\frac{1}{2} \int_{B_{i} \backslash \Omega_{t}}\left|\nabla \varphi-\omega \nabla^{\perp} X\right|^{2} \geq \pi\left|d_{i}\right||\log \varepsilon|(1-o(1)) .
$$

Hence,

$$
\begin{aligned}
\frac{1}{2} \int_{B_{i} \backslash \Omega_{t}}|\nabla U|^{2} & =\frac{1}{2} \int_{B_{i} \backslash \Omega_{t}} \eta^{4}\left|\nabla \varphi-\omega \nabla^{\perp} X\right|^{2} \\
& =\frac{1}{2} \int_{B_{i} \backslash \Omega_{t}}\left|\nabla \varphi-\omega \nabla^{\perp} X\right|^{2}+\frac{1}{2} \int_{B_{i} \backslash \Omega_{t}}\left(\eta^{4}-1\right)\left|\nabla \varphi-\omega \nabla^{\perp} X\right|^{2} .
\end{aligned}
$$

Since $0 \leq 1-\rho \leq o(1)$ on $\Omega^{\prime}$ and $|1-| v|| \leq \frac{1}{|\log \varepsilon|}$ on $\Omega^{\prime} \backslash \Omega_{t}$, (5.13) becomes

$$
\frac{1}{2} \int_{B_{i} \backslash \Omega_{t}}|\nabla U|^{2} \geq \frac{1}{2} \int_{B_{i} \backslash \Omega_{t}}\left|\nabla \varphi-\omega \nabla^{\perp} X\right|^{2}(1-o(1)) \geq \pi\left|d_{i}\right||\log \varepsilon|(1-o(1)) .
$$

From this construction, we can define the vorticity measures $\mu_{\varepsilon}$

$$
\mu_{\varepsilon}=\frac{2 \pi}{\omega} \sum_{i} d_{i} \delta_{a_{i}}
$$

where the $\left(a_{i}, d_{i}\right)$ 's are given by Proposition 5.2.

Lemma 5.1. Under the same hypotheses, we can extract a sequence $\varepsilon_{n} \rightarrow 0$ such that

$$
\begin{array}{ll}
\frac{U_{\varepsilon_{n}}}{\omega} \rightarrow U_{0} & \text { weakly in } H_{0}^{1}(\Omega), \\
& \mu_{\varepsilon_{n}} \rightarrow \mu_{0} \quad \text { in } \mathcal{R} .
\end{array}
$$

In addition,

$$
\Delta U_{0}=\mu_{0}-2
$$

Proof.

Step 1: From the upper bound of Proposition 5.1,

$$
J\left(u_{\varepsilon}\right)-F(\rho) \leq C \omega^{2} .
$$


On the other hand, as in (4.20),

$$
J\left(u_{\varepsilon}\right) \geq J(\rho)-\int_{\Omega} \frac{\omega^{2} r^{2}}{2}+\frac{1}{2} \int_{\Omega} \rho^{2}\left|\nabla v-i \omega v \nabla^{\perp} X\right|^{2} .
$$

In addition,

$$
J(\rho)-\int_{\Omega} \frac{\omega^{2} r^{2}}{2}=F(\rho)+\int_{\Omega} \frac{\omega^{2} r^{2}}{2}\left(\rho^{2}-1\right) .
$$

Since we know that $\frac{1}{\varepsilon^{2}} \int_{\Omega}\left(1-\rho^{2}\right)^{2} \leq \frac{C}{\varepsilon}$, we have $\left|\int_{\Omega} \frac{1}{2} \omega^{2} r^{2}\left(\rho^{2}-1\right)\right| \leq C \omega^{2} \varepsilon^{\frac{1}{2}}=o(1)$ because we are in the case $\omega=O(|\log \varepsilon|)$. Thus, we can transform (5.17) into

$$
J\left(u_{\varepsilon}\right) \geq F(\rho)+\frac{1}{2} \int_{\Omega} \rho^{2}|\nabla| v||^{2}+\frac{\rho^{2}|v|^{2}}{\eta^{4}}|\nabla U|^{2}-o(1) .
$$

Since $\eta \leq 1$,

$$
J\left(u_{\varepsilon}\right)-F(\rho) \geq \frac{1}{2} \int_{\Omega} \frac{|\nabla U|^{2}}{\eta^{2}}-o(1) \geq \frac{1}{2} \int_{\Omega}|\nabla U|^{2}-o(1) .
$$

Comparing to (5.16) and (5.18), we see that $\int_{\Omega}|\nabla U|^{2} \leq C \omega^{2}$, hence $\frac{U}{\omega}$ is bounded in $H_{0}^{1}$, and extracting a subsequence $\varepsilon_{n} \rightarrow 0$, we can assume that it converges weakly to some $U_{0}$ in $H_{0}^{1}$.

Step 2: We prove the same result for $\mu_{\varepsilon}$. From (5.18),

$$
C \omega^{2} \geq J(u)-F(\rho) \geq \frac{1}{2} \sum_{i \in I_{\varepsilon}} \int_{B_{i}}|\nabla U|^{2}-o(1) \geq \pi \sum_{i \in I_{\varepsilon}}\left|d_{i}\right||\log \varepsilon|(1-o(1)) \geq\left(\frac{1}{2}\left|\mu_{\varepsilon}\right|\right) \omega|\log \varepsilon|(1-o(1)) .
$$

Hence, $\int_{\Omega}\left|\mu_{\varepsilon}\right| \leq \frac{C \omega}{|\log \varepsilon|} \leq \frac{C}{\lambda}$. Since we have assumed $\lambda>0,\left(\mu_{\varepsilon}\right)$ is bounded in the sense of measures; hence, extracting again if necessary, we can assume that it converges weakly to some Radon measure $\mu_{0}$.

Step 3: There remains to compare $U_{0}$ and $\mu_{0}$. For that purpose we use the following lemma, in the spirit of Lemma 3.4 .

\section{Lemma 5.2.}

$$
\frac{1}{\omega} \operatorname{curl}(i u, \nabla u)-\mu_{\varepsilon} \rightarrow 0 \text { in } \mathcal{D}^{\prime}
$$

Proof. Consider $\xi \in C_{0}^{\infty}(\Omega)$. Since $\xi$ has compact support included in $\Omega$, for $\varepsilon$ sufficiently small, its support is included in $\Omega^{\prime}=\left\{x \in \Omega / \delta(x) \leq \varepsilon^{\beta}\right\}$. Hence, for $\varepsilon$ small enough,

$$
\int_{\Omega} \frac{\xi}{\omega} \operatorname{curl}(i u, \nabla u)=\int_{\Omega^{\prime}} \frac{\xi}{\omega} \operatorname{curl}(i u, \nabla u)
$$

and then we can proceed as in [20], Lemma 2.3 (or [3] Lem. 2.2), and obtain

$$
\int_{\Omega} \frac{\xi}{\omega} \operatorname{curl}(i u, \nabla u)=\frac{2 \pi}{\omega} \sum_{i \in I_{\varepsilon}} d_{i} \xi\left(a_{i}\right)+o(1)\|\nabla \xi\|_{L^{\infty}}
$$

Returning to the proof of the proposition, since $\mu_{\varepsilon} \rightarrow \mu_{0}$ in $\mathcal{R}$, Lemma 5.2 implies that $\frac{1}{\omega} \operatorname{curl}(i u, \nabla u) \rightarrow \mu_{0}$ in $\mathcal{D}^{\prime}$. Back to (1.10), we have

$$
\nabla^{\perp} U=(i u, \nabla u)-|u|^{2} \omega \nabla^{\perp} X
$$


Let again $\xi$ be some test-function in $C_{0}^{\infty}(\Omega)$.

$$
\left|\int_{\Omega}\left(1-|u|^{2}\right) \nabla^{\perp} X . \nabla^{\perp} \xi\right| \leq\left. C \int_{\Omega}|1-| u\right|^{2} \mid \leq C\left(\varepsilon^{2} J(u)\right)^{\frac{1}{2}} \leq o(1),
$$

from the a priori bound $J(u) \leq F(\rho)+O\left(\omega^{2}\right) \leq \frac{C}{\varepsilon}$. Hence, curl $\left(\left(1-|u|^{2}\right) \nabla^{\perp} X\right) \rightarrow 0$ in $\mathcal{D}^{\prime}$. Then, we divide (5.20) by $\omega$ and take the curl:

$$
\begin{aligned}
& \frac{\Delta U}{\omega}=\frac{\operatorname{curl}(i u, \nabla u)}{\omega}+\operatorname{curl}\left(\left(1-|u|^{2}\right) \nabla^{\perp} X\right)-\Delta X \\
& \frac{\Delta U}{\omega}=-2+\frac{\operatorname{curl}(i u, \nabla u)}{\omega}+\operatorname{curl}\left(\left(1-|u|^{2}\right) \nabla^{\perp} X\right) .
\end{aligned}
$$

We saw that $\operatorname{curl}\left(\left(1-|u|^{2}\right) \nabla^{\perp} X\right) \rightarrow 0$ in $\mathcal{D}^{\prime}, \frac{\operatorname{curl}(i u, \nabla u)}{\omega} \rightarrow \mu_{0}$ in $\mathcal{D}^{\prime}$, and $\frac{\Delta U}{\omega} \rightarrow \Delta U_{0}$ in $\mathcal{D}^{\prime}$, hence passing to the limit in (5.21), we are led to

$$
\Delta U_{0}=\mu_{0}-2
$$

\subsection{Lower bound for the energy}

We are now in a position to deduce:

Lemma 5.3. Under the same hypotheses,

$$
\liminf _{n \rightarrow \infty} \frac{J\left(u_{\varepsilon_{n}}\right)-F(\rho)}{\omega^{2}} \geq \frac{\lambda}{2} \int_{\Omega}\left|\mu_{0}\right|+\frac{1}{2} \int_{\Omega}\left|\nabla U_{0}\right|^{2}+E\left(U_{0}\right)
$$

Proof: In the spirit of [22], we start with the lower bound of (5.18):

$$
J\left(u_{\varepsilon}\right)-F(\rho) \geq \frac{1}{2} \int_{\Omega}|\nabla U|^{2}-o(1),
$$

and split the energy between that contained in the vortex-balls $B_{i}$ and that contained outside the $B_{i}$ 's:

$$
J\left(u_{\varepsilon}\right)-F(\rho) \geq \sum_{i \in I_{\varepsilon}} \frac{1}{2} \int_{B_{i}}|\nabla U|^{2}+\int_{\Omega \backslash \cup_{i} B_{i}}|\nabla U|^{2}-o(1) .
$$

Arguing as in [22], we can extract from $\varepsilon_{n}$ a subsequence if necessary and define

$$
\mathcal{A}_{N}=\cup_{n>N} \cup_{i \in I_{\varepsilon_{n}}} B_{i}
$$

such that meas $\mathcal{A}_{N} \rightarrow 0$ as $N \rightarrow \infty$, and $\mathcal{A}_{N}$ contains all the balls. Then, by weak convergence of $\frac{U}{\omega}$ to $U_{0}$,

$$
\liminf _{n \rightarrow \infty} \frac{1}{\omega^{2}} \int_{\Omega \backslash \cup_{i} B_{i}}|\nabla U|^{2} \geq \liminf _{n \rightarrow \infty} \frac{1}{\omega^{2}} \int_{\Omega \backslash \mathcal{A}_{N}}|\nabla U|^{2} \geq \int_{\Omega \backslash \mathcal{A}_{N}}\left|\nabla U_{0}\right|^{2} .
$$

On the other hand, from (5.18),

$$
\sum_{i \in I_{\varepsilon_{n}}} \frac{1}{2} \int_{B_{i}} \frac{|\nabla U|^{2}}{\omega^{2}} \geq \frac{\left|\log \varepsilon_{n}\right|}{2 \omega} \int_{\Omega}\left|\mu_{\varepsilon_{n}}\right|
$$


and, by convexity of $\mu \mapsto \int_{\Omega}|\mu|$ and weak convergence of $\mu_{\varepsilon_{n}}$,

$$
\liminf _{n \rightarrow \infty} \frac{1}{2 \omega^{2}} \sum_{i \in I_{\varepsilon_{n}}} \frac{1}{2} \int_{B_{i}}|\nabla U|^{2} \geq \frac{\lambda}{2} .
$$

Combining (5.23-5.25), we are led to

$$
\liminf \frac{J(u)-F(\rho)}{\omega^{2}} \geq \frac{\lambda}{2} \int_{\Omega}\left|\mu_{0}\right|+\frac{1}{2} \int_{\Omega \backslash \mathcal{A}_{N}}\left|\nabla U_{0}\right|^{2} .
$$

Since this is true for all $N$, passing to the limit as $N \rightarrow \infty$, we obtain

$$
\liminf \frac{J(u)-F(\rho)}{\omega^{2}} \geq \frac{\lambda}{2} \int_{\Omega}\left|\mu_{0}\right|+\frac{1}{2} \int_{\Omega}\left|\nabla U_{0}\right|^{2} .
$$

Therefore, we have the lower bound

$$
\liminf _{n \rightarrow \infty} \frac{J\left(u_{\varepsilon_{n}}\right)-F(\rho)}{\omega^{2}} \geq \inf E
$$

corresponding to the upper bound of Proposition 5.1. There only remains to check that $\min E$ is achieved, and what the minimizer is.

Proposition 5.3. For any $\lambda \geq 0, \min _{V} E$ is uniquely achieved by a $U_{*} \in H_{0}^{1} \cap C^{0, \gamma}(\forall \gamma<1)$, solution of the following obstacle problem (we write $\mu_{*}=\Delta U_{*}+2$ ):

$$
\begin{cases}U_{*}=0 & \text { on } \partial \Omega \\ U_{*} \leq \frac{\lambda}{2} & \text { in } \Omega \\ \left(\Delta U_{*}+2\right)\left(U_{*}-\frac{\lambda}{2}\right)=0 & \text { in } \Omega .\end{cases}
$$

Moreover $\mu_{*} \geq 0$ and $\mu_{*} \in L^{\infty}(\Omega)$. In addition, if we define the set $\mathcal{U}_{\lambda}$ to be $\mathcal{U}_{\lambda}=S u p p \mu_{*}=\left\{x \in \Omega / U_{*}(x)\right.$ $\left.=\frac{\lambda}{2}\right\}$, then $\mathcal{U}_{\lambda}=\varnothing \Leftrightarrow \lambda>2 \max \xi_{0}$ where $\xi_{0}$ is given by (1.16).

Proof. The minimum of $E$ is achieved by some $U_{*}$ by weak lower semi-continuity, and this minimizer is unique since $E$ is convex. We observe that for any $f \in H_{0}^{1}$ with $\mu=\Delta f+2 \in \mathcal{R}$,

$$
f(y)=-\int_{\Omega} G(x, y) d(\mu(x)-2) \quad \text { a.e. in } y .
$$

(We recall that $-\Delta_{x} G=\delta_{y}$.) Thus

$$
E(f)=\frac{\lambda}{2} \int_{\Omega}|\mu|+\frac{1}{2} \int_{\Omega \times \Omega} G(x, y) d(\mu(x)-2) d(\mu(y)-2) .
$$

Making variations in $\mu$ around $\mu_{*}=\Delta U_{*}+2$ as in [22], we obtain that

$$
\begin{aligned}
& \frac{\lambda}{2}\left|\mu_{*}\right|-U_{*} \mu_{*}=0, \\
& -\frac{\lambda}{2} \leq U_{*} \leq \frac{\lambda}{2} \quad \text { a.e. }
\end{aligned}
$$


From (5.29), we deduce, denoting by $\mu_{*}^{+}$and $\mu_{*}^{-}$the positive and negative parts of $\mu_{*}\left(\mu_{*}=\mu_{*}^{+}-\mu_{*}^{-}\right)$, that

$$
\left\{\begin{array}{lll}
U_{*}=\frac{\lambda}{2} & \mu_{*}^{+} \text {a.e. } \\
U_{*}=-\frac{\lambda}{2} & \mu_{*}^{-} \text {a.e. }
\end{array}\right.
$$

Denoting by $U_{*}^{-}$the negative part of $U_{*}$,

$$
\int_{\Omega} \mu_{*} U_{*}^{-}=\int_{\Omega}\left(\Delta U_{*}+2\right) U_{*}^{-}=-\int_{\Omega}\left|\nabla U_{*}\right|^{2}+2 \int_{\Omega} U_{*} \leq 0 .
$$

On the other hand,

$$
\int_{\Omega} \mu_{*} U_{*}=\int_{\Omega} U_{*}^{-} \mu_{*}^{+}-\int_{\Omega} U_{*}^{-} \mu_{*}^{-}
$$

Since $U_{*}=\frac{\lambda}{2}, \mu_{*}^{+}$- almost everywhere, there remains

$$
\int_{\Omega} \mu_{*} U_{*}^{-}=\frac{\lambda}{2} \int_{\Omega} \mu_{*}^{-} \geq 0
$$

Combining this with (5.31), we have $\int_{\Omega}\left|\mu_{*}^{-}\right|=0$ hence $\mu_{*}=\mu_{*}^{+}$is a positive measure. Thus, $U_{*}$ satisfies

$$
\left\{\begin{array}{l}
U_{*} \leq \frac{\lambda}{2} \\
U_{*}=0 \\
\mu_{*} \geq 0 \\
\mu_{*}\left(U_{*}-\frac{\lambda}{2}\right)=0 .
\end{array} \quad \text { on } \partial \Omega\right.
$$

The proof of [22] adjusts also easily to state that $\mu_{*}$ is absolutely continuous with respect to the Lebesgue measure, and in fact $L^{\infty}$, hence $U_{*} \in C^{0, \gamma}(\forall \gamma<1)$.

If we denote by $\mathcal{U}_{\lambda}$ the "vortex-region", i.e. the support of $\mu_{*}$, or the set $\left\{x \in \Omega / U_{*}(x)=\frac{\lambda}{2}\right\}$, we prove that $\mathcal{U}_{\lambda}=\varnothing \Leftrightarrow \lambda>2 \max \xi_{0}$. This comes from the maximum principle: recall that $\xi_{0}$ is the solution of

$$
\begin{cases}-\Delta \xi_{0}=2 & \text { in } \Omega \\ \xi_{0}=0 & \text { on } \partial \Omega\end{cases}
$$

Observe that $\xi_{0}=\frac{R^{2}}{2}-\frac{|x|^{2}}{2}$ if $\Omega=B(0, R) . \mu_{*} \geq 0$ means that $\Delta U_{*} \geq 2$ hence $-\Delta\left(\xi_{0}-U_{*}\right) \geq 0$. In addition, $\xi_{0}-U_{*}=0$ on $\partial \Omega$; consequently, by the maximum principle, $\xi_{0} \geq U_{*}$ in $\Omega$. Now if $\mathcal{U}_{\lambda} \neq \varnothing$, there exists $x \in \Omega$ such that $U_{*}(x)=\frac{\lambda}{2}$ and $\xi_{0}(x) \geq \frac{\lambda}{2}$. Therefore, $\lambda \leq 2 \max \xi_{0}$. On the other hand, if $\mathcal{U}_{\lambda}=\varnothing$, then the equation for $U_{*}$ reduces to

$$
\begin{cases}U_{*} \leq \frac{\lambda}{2} & \\ U_{*}=0 & \text { on } \partial \Omega \\ \Delta U_{*}+2=0 & \text { in } \Omega .\end{cases}
$$

Hence $U_{*}=\xi_{0}$ and $\xi_{0} \leq \frac{\lambda}{2}$ and $\lambda \geq 2 \max \xi_{0}$.

Comparing the upper bound of Proposition 5.1 to the lower bound of Lemma 5.3, we have

$$
E\left(U_{*}\right) \geq \liminf _{n \rightarrow \infty} \frac{J\left(u_{\varepsilon_{n}}\right)-F(\rho)}{\omega^{2}} \geq E\left(U_{0}\right) \geq E\left(U_{*}\right) .
$$

Hence, by uniqueness of the minimizer of $E, U_{0}=U_{*}$ and $\mu_{0}=\mu_{*}$. Furthermore, these are the only possible limits for sequences extracted from $U_{\varepsilon}$ and $\mu_{\varepsilon}$, hence the whole families $U_{\varepsilon}$ and $\mu_{\varepsilon}$ converge to $U_{*}$ and $\mu_{*}$. 
We have thus proved Theorem 3 in the case $\lambda>0$. In the case $\lambda=0$, returning to (4.20), we have

$$
\begin{aligned}
J(u) & \geq J(\rho)-\frac{1}{2} \int_{\Omega} \omega^{2} r^{2}+\frac{1}{2} \int_{\Omega} \rho^{2}|v|^{2} \frac{|\nabla U|^{2}}{\eta^{4}} \geq F(\rho)+\frac{\omega^{2}}{2} \int_{\Omega} r^{2}\left(\rho^{2}-1\right)+\frac{1}{2} \int_{\Omega}|\nabla U|^{2} \\
& \geq F(\rho)+\frac{1}{2} \int_{\Omega}|\nabla U|^{2}+o\left(\omega^{2}\right),
\end{aligned}
$$

since $\int_{\Omega}\left|1-\rho^{2}\right|=o(1)$. On the other hand, we saw in Proposition 4.1 that for $\omega \ll \frac{1}{\varepsilon^{2}}$ we have the upper bound $\min J \leq F(\rho)+o\left(\omega^{2}\right)$, hence if $u$ minimizes $J, \frac{1}{2} \int_{\Omega}|\nabla U|^{2} \leq o\left(\omega^{2}\right)$. We conclude that $\frac{U}{\omega} \rightarrow 0$ in $H_{0}^{1}(\Omega)$, and that the assertions of Theorem 3 remain true in the case $\lambda=0, \omega \ll \frac{1}{\varepsilon^{2}}$.

The author is grateful to Yves Pomeau for suggesting the problem and for very interesting discussions, and to Itai Shafrir for his helpful suggestions.

\section{REFERENCES}

[1] L. Almeida and F. Bethuel, Topological Methods for the Ginzburg-Landau Equations. J. Math. Pures Appl. 77 (1998) 1-49.

[2] A. Aftalion (in preparation.)

[3] A. Aftalion, E. Sandier and S. Serfaty, Pinning Phenomena in the Ginzburg-Landau Model of Superconductivity. J. Math. Pures Appl. (to appear).

[4] N. André and I. Shafrir, Minimization of a Ginzburg-Landau type functional with nonvanishing Dirichlet boundary condition. Calc. Var. Partial Differential Equations (1998) 1-27.

[5] F. Bethuel, H. Brezis and F. Hélein, Ginzburg-Landau Vortices. Birkhäuser (1994).

[6] A. Bonnet and R. Monneau, Distribution of vortices in a type-II superconductor as a free boundary problem: Existence and regularity via Nash-Moser theory. Interfaces Free Bound. 2 (2000) 181-200.

[7] H. Brezis and L. Oswald, Remarks on sublinear elliptic equations. Nonlinear Anal. 10 (1986) 55-64.

[8] D.A. Butts and D.S. Rokhsar, Predicted signatures of rotating Bose-Einstein condensates. Nature 397 (1999) $327-329$.

[9] Y. Castin and R. Dum, Bose-Einstein condensates with vortices in rotating traps. Eur. Phys. J. D 7 (1999) 399-412.

[10] A. Fetter, Vortices and Ions in Helium, in The physics of liquid and solid helium, part I, edited by K.H. Bennemann and J.B. Keterson. John Wiley, Interscience, Interscience Monographs and Texts in Physics and Astronomy 30 (1976).

[11] S. Gueron and I. Shafrir, On a Discrete Variational Problem Involving Interacting Particles. SIAM J. Appl. Math. 60 (2000) $1-17$.

[12] D. Kinderlehrer and G. Stampacchia, An introduction to variational inequalities and their applications. Acad. Press (1980).

[13] L. Lassoued and P. Mironescu, Ginzburg-Landau type energy with discontinuous constraint. J. Anal. Math. 77 (1999) 1-26.

[14] N. Owen, J. Rubinstein and P. Sternberg, Minimizers and gradient flows for singularly perturbed bi-stable potentials with a Dirichlet condition. Proc. Roy. Soc. London Ser. A 429 (1990) 503-532.

[15] J.F. Rodrigues, Obstacle Problems in Mathematical Physics. Mathematical Studies, North Holland (1987).

[16] S. Serfaty, Local Minimizers for the Ginzburg-Landau Energy near Critical Magnetic Field, Part I. Comm. Contemporary Math. 1 (1999) 213-254.

[17] S. Serfaty, Local Minimizers for the Ginzburg-Landau Energy near Critical Magnetic Field, Part II. Comm. Contemporary Math. 1 (1999) 295-333.

[18] S. Serfaty, Stable Configurations in Superconductivity: Uniqueness, Multiplicity and Vortex-Nucleation. Arch. Rational Mech. Anal. 149 (1999) 329-365.

[19] S. Serfaty, Sur l'équation de Ginzburg-Landau avec champ magnétique, in Proc. of Journées Équations aux dérivées partielles, Saint-Jean-de-Monts (1998).

[20] E. Sandier and S. Serfaty, Global Minimizers for the Ginzburg-Landau Functional below the First Critical Magnetic Field. Ann. Inst. H. Poincaré Anal. Non Linéaire 17 (2000) 119-145.

[21] E. Sandier and S. Serfaty, On the Energy of Type-II Superconductors in the Mixed Phase. Rev. Math. Phys. (to appear).

$[22]$ E. Sandier and S. Serfaty, A Rigorous Derivation of a Free-Boundary Problem Arising in Superconductivity. Annales Sci. École Norm. Sup. (4) 33 (2000) 561-592.

[23] E. Sandier and S. Serfaty, Ginzburg-Landau Minimizers Near the First Critical Field Have Bounded Vorticity. Preprint.

[24] D. Tilley and J. Tilley, Superfluidity and Superconductivity, 2nd edition. Adam Hilger Ltd., Bristol (1986). 